\title{
A Stochastic Generalized Ginzburg-Landau Equation Driven by Jump Noise
}

\author{
Lin Lin* and Hongjun Gao ${ }^{\dagger}$
}

\begin{abstract}
This paper is concerned with the stochastic generalized Ginzburg-Landau equation driven by a multiplicative noise of jump type. By a prior estimate, weak convergence and monotonicity technique, we prove the existence and uniqueness of the solution of an initialboundary value problem with homogeneous Dirichlet boundary condition. However, for the generalized Ginzburg-Landau equation, such a locally monotonic condition of the nonlinear term can not be satisfied in a straight way. For this, we utilize the characteristic structure of nonlinear term and refined analysis to overcome this gap.
\end{abstract}

Key words: stochastic generalized Ginzburg-Landau equation, jump noise, existence and uniqueness.

Mathematics Subject Classification: 60H15, 35Q99

\section{Introduction}

The deterministic Ginzburg-Landau partial differential equation had been used to model phenomena in a number of different areas in physics and other fields [12, 13, 24], and it was extended to the generalized Ginzburg-Landau equation with derivative nonlinear term by Doelman in [6]. Many results on the existence and uniqueness were studied under various assumptions on the parameters [9, 10, 11, 12, 13, 15. However, some perturbations may be neglected in the derivation of this ideal model. Researchers often represent the microscopic effects by random perturbations in the dynamics of the macroscopic observables. Thus, it is natural to consider stochastic effect in the Ginzburg-Landau equation. Recently, the stochastic Ginzburg-Landau equation with additive or multiplicative Gaussian noise has been studied by a few authors (see, e.g. [25, 26, 27, 28]), among which Yang [27, 28] considered large deviations for the generalized 1-D stochastic Ginzburg-Landau equation and existence for the generalized 2-D stochastic Ginzburg-Landau equation with multiplicative Gaussian noise.

As it is well known, most of the works of the stochastic generalized Ginzburg-Landau equation are driven by Gaussian white noise, but stochastic partial differential equations driven by jump noise have important applications in mathematical physics [2, 23]. The applied backgrounds of jump noise is also our main motivation to consider the GinzburgLandau equation driven by jump noise. There are lots of existing results related to

\footnotetext{
* Jiangsu Provincial Key Laboratory for NSLSCS, School of Mathematical Sciences, Nanjing Normal University, Nanjing 210023, China. (Email: linlin@njnu.edu.cn)

${ }^{\dagger}$ Corresponding author. School of Mathematical Sciences and Jiangsu Center for Collaborative Innovation in Geographical Information Resource Development and Application, Nanjing Normal University, Nanjing 210023, China. (Email: gaohj@njnu.edu.cn, hjgao-nj@163.com)
} 
stochastic partial differential equations driven by jump noise, such as [3, 4, 5, 7, 8, 16, 21, 22]. To be specific, Brzeźniak and Zhu [3] obtained the existence and uniqueness of solutions for a type of stochastic nonlinear beam equations with Poisson-type noises; Brzeźniak [4] considered the 2-D stochastic Navier-Stokes equations driven by jump noise; and Sun and Gao [22] studied the well-posedness for 2-D stochastic Primitive equations with Lévy noise.

In Brzeźniak and Liu [5], the authors built a unified framework for SPDE with locally monotonic coefficients driven by Lévy noise, in which the existence and uniqueness of the solution in a fixed probability space is proved based on a prior estimate, weak convergence and monotonicity arguments as in [20]. However, this method does not work for the generalized Ginzburg-Landau equation since the local monotonicity of the nonlinear operator cannot be satisfied in a straight way. In order to obtain the local monotonicity in (3.27), we first use the nonlinear structure and refined analysis (such as the analy-

sis of $\left.\int_{0}^{T} \int_{D}\left|u_{n}\right|^{2 \sigma}\left|\nabla u_{n}\right|^{2} d x d t\right)$ to overcome the gaps. Also the uniform bounds for $u_{n}$ in $L^{p}\left(\Omega, L^{\infty}([0, T], V)\right)$ is established for some $p \geq 2$ (Lemma 3.3), which is important to enable $r(t)$ in (3.28) to make sense. Moreover, different from the deterministic case in which $\operatorname{Re} I \leq 0$ is enough, the more elaborate estimate $\operatorname{Re} I \leq-C\left\|u_{n}-\phi\right\|_{2 \sigma+2}^{2 \sigma+2}, C>0$ is needed (Lemma 3.5), which is helpful for the estimates of some items of Re $J$ in Lemma $3.6(I, J$ are defined in Section 3).

In this paper, we will study the following stochastic generalized Ginzburg-Landau equation (SGGLE) driven by jump noise:

$$
\left\{\begin{array}{lc}
d u=\left((1+i \alpha) \Delta u-(1-i \beta)|u|^{2 \sigma} u+\gamma u+F(u)\right) d t+\int_{Z} g(t, u, z) \tilde{\eta}(d z, d t), & t \geq 0, x \in \partial D, \\
u(x, t)=0, & x \in D,
\end{array}\right.
$$

where $D=\left(0, L_{1}\right) \times\left(0, L_{2}\right), i=\sqrt{-1}, \gamma>0$, the parameters $\alpha, \beta$ are all real-valued constants and $u$ is a complex-valued scalar function. The derivative term $F(u)=\lambda_{1}$. $\nabla\left(|u|^{2} u\right)+\left(\lambda_{2} \cdot \nabla u\right)|u|^{2}$ with two complex constant vectors $\lambda_{1}$ and $\lambda_{2} . \tilde{\eta}$ is the Lévy process defined on a complete probability space and $g$ is a given function which will be defined later. For the deterministic 2-D generalized Ginzburg-Landau equation, Li and Guo [15] proved that the equation has a unique solution under the following assumptions on the parameters $\alpha, \beta$ and $\sigma$ :

(1) Either (a) $\sigma>2$ or (b) $\sigma=2$ and $\left|\lambda_{i}\right|, i=1,2$, are sufficiently small;

(2) $-1+\alpha \beta<\frac{\sqrt{2 \sigma+1}}{\sigma}|\alpha+\beta|$.

The next two sections are organized as follows. In Section 2, we recall some fundamental concepts related to Lévy process and present the main theorem. In section 3, we use the Galerkin method to prove the main theorem, i.e. existence and uniqueness of solution to our concerned equation.

\section{Preliminaries and main theorem}

Firstly, we introduce some definitions and basic properties of Lévy processes. The readers can also refer to [19] for more details. 
Let $(\Omega, \mathscr{F}, \mathbb{F}, \mathbb{P})$ be a filtered probability space, where $\mathbb{F}=\left(\mathscr{F}_{t}\right)_{t \geq 0}$ is a filtration, $(Z, \mathcal{Z})$ be a measurable space, and $\nu$ be a $\sigma$-finite positive measure on it. We denote the Borel $\sigma$-field on a topological space X by $\mathcal{B}(X)$. Let $\eta: \mathscr{F} \times \mathcal{B}\left(R^{+}\right) \times \mathcal{Z} \rightarrow \overline{\mathbb{N}}=\mathbb{N} \cup\{\infty\}$ be a time homogeneous Poisson random measure with the intensity measure $\nu$ defined over $(\Omega, \mathscr{F}, \mathbb{F}, \mathbb{P})$. We denote by $\tilde{\eta}(d t, d z)=\eta(d t, d z)-d t \nu(d z)$ the compensated Poisson random measure associated to $\eta$.

Suppose that $\left(H,|\cdot|_{H}\right)$ is a Hilbert space. We then define a unique continuous linear operator $I$ serving as the stochastic integration with respect to the $\mathbb{F}$-predictable process $\xi:[0, T] \times Z \times \Omega \rightarrow H$ with

$$
\mathbb{E} \int_{0}^{T} \int_{Z}|\xi(r, z)|_{H}^{2} \nu(d z) d r<\infty, \quad T>0
$$

For any random step process $\xi$ satisfying the condition (2.1) with a representation

$$
\xi(r)=\sum_{j=1}^{n} 1_{\left(t_{j-1}, t_{j}\right]}(r) \xi_{j}, \quad r \geq 0
$$

where $\left\{0=t_{0}<t_{1}<\ldots<t_{n}<\infty\right\}$ is a partition of $[0, \infty)$ and $\xi_{j}$ is an $\mathscr{F}_{t_{j-1}}$ measurable random variable for $j=1,2, \cdots, n$, we define $I(\xi)$ to be an $H$-valued adapted and càdlàg process as follows

$$
I(\xi)(t)=\sum_{j=1}^{n} \int_{Z} \xi_{j}(z) \tilde{\eta}\left(d z,\left(t_{j-1} \wedge t, t_{j} \wedge t\right]\right), \quad t \geq 0 .
$$

Usually, we write

$$
I(\xi)(t):=\int_{0}^{t} \int_{Z} \xi(r, z) \tilde{\eta}(d r, d z), \quad t \geq 0 .
$$

The continuity of the operator $I$ means that

$$
\mathbb{E}\left|\int_{0}^{t} \int_{Z} \xi(r, z) \tilde{\eta}(d r, d z)\right|_{H}^{2}=\mathbb{E} \int_{0}^{t} \int_{Z}|\xi(r, z)|_{H}^{2} \nu(d z) d r, \quad t \geq 0 .
$$

For fixed $T>0$, we denote by $\mathcal{M}^{2}\left(0, T ; L^{2}(Z, \nu ; H)\right)$ the class of all $\mathbb{F}$-predictable processes $\xi:[0, T] \times Z \times \Omega \rightarrow H$ satisfying the condition (2.1), where $L^{2}(Z, \nu ; H)$ is defined as the class of all functions $\eta: Z \rightarrow H$ satisfying $\int_{Z}|\eta(z)|_{H}^{2} \nu(d z)<\infty$.

Before giving the proof of the existence and uniqueness of solution, we need clarify some useful lemmas.

Itô formula in Hilbert space is needed in our situation and one can refer to Itô formula for semimartingale in [17] (Theorem 27.2) for $\phi: E \rightarrow G$ be a $C^{2}$ function or the following one for the process given by (2.2) below in [29] (Theorem 3.5.3).

Lemma 2.1 Assume that $E$ is an Hilbert space. Let $X$ be a process given by

$$
X_{t}=X_{0}+\int_{0}^{t} a(s) d s+\int_{0}^{t} \int_{Z} f(s, z) \tilde{\eta}(d s, d z), \quad t \geq 0
$$


where $a$ is an $E$-valued progressively measurable process on the space $\left(\mathbb{R}_{+} \times \Omega, \mathcal{B}_{\mathbb{R}_{+}} \times \mathscr{F}\right)$ such that for all $t \geq 0, \int_{0}^{t}\|a(s, w)\|_{E} d s<\infty$ a.s. and $f$ is a predictable process on $E$ with $\mathbb{E} \int_{0}^{T} \int_{Z}|f(s, z)|^{2} \nu(d z) d s<\infty$. Let $G$ be a separable Hilbert space and $\phi: E \rightarrow G$ be a function of $C^{1}$ such that $\phi^{\prime}$ is Hölder continuous. Then for each $t>0$, we have $\mathbb{P}$-a.s.

$$
\begin{aligned}
\phi\left(X_{t}\right)= & \phi\left(X_{0}\right)+\int_{0}^{t} \phi^{\prime}\left(X_{s}\right) a(s) d s+\int_{0}^{t} \int_{Z}\left[\phi^{\prime}\left(X_{s-}\right) f(s, z)\right] \tilde{\eta}(d s, d z) \\
& +\int_{0}^{t} \int_{Z}\left[\phi\left(X_{s-}+f(s, z)\right)-\phi\left(X_{s-}\right)-\phi^{\prime}\left(X_{s-}\right) f(s, z)\right] \eta(d z d s) .
\end{aligned}
$$

Lemma 2.2 (Burkholder-Davis-Gundy Inequality [14]) For any $p \geq 2$ and $T>0$, there exists a constant $C_{p}$ such that for any real-valued square integrable càdlàg martingale $M$ with $M(0)=0$, we have

$$
\sup _{0 \leq t \leq T}|M(t)|^{p} \leq C_{p} \mathbb{E}[M]_{T}^{p / 2},
$$

where $[M]_{t}, 0 \leq t \leq T$, is the Meyer process of $M$.

Lemma 2.3 (Okazawa and Yokota [18]) Let $H$ be a complex Hilbert space with inner product $\langle\cdot, \cdot\rangle$ and norm $\|\cdot\|_{H}$. For $p \in(1, \infty)$ and any non-zero $z, w \in H$ with $z \neq w$, we have the following inequality:

$$
\frac{\left|\operatorname{Im}\left\langle\|z\|_{H}^{p-2} z-\|w\|_{H}^{p-2} w, z-w\right\rangle\right|}{\operatorname{Re}\left\langle\|z\|_{H}^{p-2} z-\|w\|_{H}^{p-2} w, z-w\right\rangle} \leq \frac{|p-2|}{2 \sqrt{p-1}} .
$$

For the mathematical setting of our problem, we introduce complex Sobolev spaces. Denote $(\cdot, \cdot)$ the inner product and the norm in $L^{2}(D)$, where

$$
(u, v)=\operatorname{Re} \int_{D} u(x) \bar{v}(x) d x,
$$

for $u, v \in L^{2}(D)$.

We always write $H=L^{2}, V=H_{0}^{1},\|\cdot\|_{p}=\|\cdot\|_{L^{p}}$ and $\|\cdot\|=\|\cdot\|_{2}$ when $p=2$ for simplicity. Let $A u=(1+i \alpha) \triangle u$ and $B u=-(1-i \beta)|u|^{2 \sigma} u+\gamma u+F(u)$. The operator $A$ is an isomorphism from $D(A)=V \cap H^{2}$ onto $H$. We now write (1.1) in the following abstract form:

$$
\left\{\begin{array}{l}
d u(t)=(A u(t)+B u(t)) d t+\int_{Z} g(t, u, z) \tilde{\eta}(d z, d t), \\
u(x, t)=0, \quad t \geq 0, x \in \partial D, \\
u(x, 0)=u_{0}(x),
\end{array}\right.
$$

To obtain the existence of solution to (2.3), we assume that the function $g:[0, \infty) \times H \rightarrow$ $L^{2}(Z, \nu ; H)$ is a measurable function and there exist nonnegative constants $k_{i}, i=1,2,3,4$ ,$h \in L^{p}(Z, \nu ; H)(2 \leq p<2 \sigma)$ such that for any $t \in[0, T]$ and all $u, v \in V$,

$\left(C_{1}\right):\|g(t, u)\|_{L^{2}(Z, \nu ; H)}^{2} \leq k_{1}\|u\|^{2}+k_{2}\|\nabla u\|^{2} ;$

$\left(C_{2}\right):\|g(t, u)-g(t, v)\|_{L^{2}(Z, \nu ; H)}^{2} \leq k_{3}\|u-v\|^{2}+k_{4}\|\nabla(u-v)\|^{2}$

$\left(C_{3}\right):\left|\partial_{u} g(t, u, z)\right| \leq h(z)|u|$ for all $z \in Z$. 
Definition 2.4 An $H$-valued càdlàg $\mathbb{F}$-adapted process $u(t)$ is said to be a solution of the stochastic generalized Ginzburg-Landau equation with jump noise (2.3) if for its $d t \times d \mathbb{P}$ equivalent class $\tilde{u}$ we have

(1) $\tilde{u} \in L^{p}\left(\Omega ; L^{\infty}([0, T] ; V)\right)$;

(2) For every $t \in[0, T]$, the following equality holds $\mathbb{P}$-a.s.:

$$
u(t)=u_{0}+\int_{0}^{t} A \tilde{u}(s) d s+\int_{0}^{t} B \tilde{u}(s) d s+\int_{0}^{t} \int_{Z} g(s, \tilde{u}(s), z) \tilde{\eta}(d z, d s) .
$$

The main result of this paper is the following theorem.

Theorem 2.5 Suppose that conditions $\left(C_{1}\right)-\left(C_{3}\right)$ hold, $2 \leq p<2 \sigma, \sigma>2$ and $0<|\beta|<\frac{\sqrt{2 \sigma+1}}{\sigma}$. Then for any $H$-valued function $u_{0}$ satisfying $\mathbb{E}\left\|\nabla u_{0}\right\|^{p}<\infty, \operatorname{SPDE}$ (2.3) has a unique solution $u=u(t), 0 \leq t \leq T$, satisfying

$$
\mathbb{E}\left(\sup _{0 \leq s \leq T}\|u(s)\|^{2}+\int_{0}^{T}\|\nabla u(s)\|^{2} d s+\int_{0}^{T}\|u(s)\|_{2 \sigma+2}^{2 \sigma+2} d s\right) \leq C\left(\mathbb{E}\left\|u_{0}\right\|^{2}+1\right)
$$

and the additional regularity

$$
u(t) \in L^{2}\left(\Omega ; L^{2}\left([0, T] ; H^{2}\right)\right) \cap L^{p}\left(\Omega ; L^{\infty}([0, T] ; V)\right) .
$$

\section{Proof of main theorem}

The main method for the proof of Theorem 2.5 is the Galerkin approximation of (2.3) and we divide the proof into four steps. The first three steps give the existence proof of solution to (2.3) and the proof of uniqueness of solution is demonstrated in step 4.

Step 1: Suppose that $\left\{e_{i}: i \in N\right\} \subset D(A)$ is an orthonormal basis of $H$ such that $\operatorname{span}\left\{e_{i}: i \in N\right\}$ is dense in $V$. Denote $H_{n}:=\operatorname{span}\left\{e_{1}, \ldots e_{n}\right\}$. Set $P_{n}: H \rightarrow H_{n}$ to be

$$
P_{n} x=\sum_{i=1}^{n}\left\langle x, e_{i}\right\rangle e_{i}
$$

where $P_{n}$ is the orthogonal projection onto $H_{n}$ in $H$.

For simplicity, we denote $G(u)=A(u)+B(u)$. For each finite $n \in N$, we consider the following equation:

$$
u_{n}(t)=u_{n}(0)+\int_{0}^{t} P_{n} G\left(u_{n}(s)\right) d s+\int_{0}^{t} \int_{Z} P_{n} g\left(s, u_{n}(s), z\right) \tilde{\eta}(d z, d s), \quad t \in[0, T],
$$

where $u_{n}(0)=P_{n} u_{0}$. According to [1], (3.1) has a unique càdlàg strong solution.

Then we give some priori estimates as preparation of proof of Theorem 2.5.

Lemma 3.1 Under the same assumptions as in Theorem 2.5, there exists a constant $C$ such that

$$
\sup _{n} \mathbb{E}\left(\sup _{0 \leq t \leq T}\left\|u_{n}(t)\right\|^{2}+\int_{0}^{T}\left\|\nabla u_{n}(s)\right\|^{2} d s+\int_{0}^{T}\left\|u_{n}(s)\right\|_{2 \sigma+2}^{2 \sigma+2} d s\right) \leq C\left(\mathbb{E}\left\|u_{0}\right\|^{2}+1\right) .
$$

Proof : Applying Itô formula to the process $\left\|u_{n}(t)\right\|^{2}$ and taking the real part, we obtain

$$
\left\|u_{n}(t)\right\|^{2}
$$




$$
\begin{aligned}
= & \left\|u_{n}(0)\right\|^{2}+2 \operatorname{Re} \int_{0}^{t}\left(u_{n}(s),(1+i \alpha) \triangle u_{n}(s)\right) d s+2 \int_{0}^{t}\left(u_{n}(s), \gamma u_{n}(s)\right) d s \\
& -2 \operatorname{Re} \int_{0}^{t}\left(u_{n}(s),(1-i \beta)\left|u_{n}(s)\right|^{2 \sigma} u_{n}(s)+F\left(u_{n}(s)\right)\right) d s \\
& +\int_{0}^{t} \int_{Z}\left\|P_{n} g\left(s, u_{n}(s), z\right)\right\|^{2} \eta(d s, d z)+2 \operatorname{Re} \int_{0}^{t} \int_{Z}\left(u_{n}(s-), P_{n} g\left(s, u_{n}(s), z\right)\right) \tilde{\eta}(d s, d z) \\
= & \left\|u_{n}(0)\right\|^{2}-2 \int_{0}^{t}\left\|\nabla u_{n}(s)\right\|^{2} d s+2 \gamma \int_{0}^{t}\left\|u_{n}(s)\right\|^{2} d s \\
& -2 \int_{0}^{t}\left\|u_{n}(s)\right\|_{2 \sigma+2}^{2 \sigma+2} d s+I_{1}(t)+I_{2}(t)+I_{3}(t),
\end{aligned}
$$

where

$$
\begin{aligned}
& I_{1}(t)=\int_{0}^{t} \int_{Z}\left\|P_{n} g\left(s, u_{n}(s), z\right)\right\|^{2} \eta(d s, d z) \\
& I_{2}(t)=2 \operatorname{Re} \int_{0}^{t} \int_{Z}\left(u_{n}(s-), P_{n} g\left(s, u_{n}(s), z\right)\right) \tilde{\eta}(d s, d z) \\
& I_{3}(t)=-2 \operatorname{Re} \int_{0}^{t}\left(u_{n}(s), \lambda_{1} \cdot \nabla\left(\left|u_{n}(s)\right|^{2} u_{n}(s)\right)+\left(\lambda_{2} \cdot \nabla u_{n}(s)\right)\left|u_{n}(s)\right|^{2}\right) d s .
\end{aligned}
$$

Now, for each natural number $R$, we consider the stopping time $\tau_{R}^{n}:=\inf \{t \geq 0$ : $\left.\left\|u_{n}(t)\right\|^{2} \geq R\right\} \wedge T$. Since the process $u_{n}(t), t \in[0, T]$, is adapted and càdlàg, it is obvious that $\tau_{R}^{n} \uparrow T$, and $\mathbb{P}\left\{\tau_{R}^{n}<T\right\}=0$ as $R \rightarrow \infty$.

First we have

$$
\begin{aligned}
\mathbb{E} \sup _{0 \leq s \leq t \wedge \tau_{R}^{n}} I_{1}(s) & =\mathbb{E} \int_{0}^{t \wedge \tau_{R}^{n}} \int_{Z}\left\|P_{n} g\left(s, u_{n}(s), z\right)\right\|^{2} \nu(d z) d s \\
& \leq k_{1} \mathbb{E} \int_{0}^{t \wedge \tau_{R}^{n}}\left\|u_{n}(s)\right\|^{2} d s+k_{2} \mathbb{E} \int_{0}^{t \wedge \tau_{R}^{n}}\left\|\nabla u_{n}(s)\right\|^{2} d s
\end{aligned}
$$

Here the first equality is due to the martingale property of the stochastic integration with repect to the compensated Poisson random measure $\tilde{\eta}(d t, d z)=\eta(d t, d z)-d t \nu(d z)$ and the second inequality is due to the application of $\left(C_{1}\right)$.

And the process $I_{2}$ is a martingale, we can apply the B-D-G inequality in Lemma 2.2 and the condition $\left(C_{1}\right)$ again to get

$$
\begin{aligned}
\mathbb{E} \sup _{0 \leq s \leq t \wedge \tau_{R}^{n}} I_{2}(s) \leq & 2 \mathbb{E} \sup _{0 \leq s \leq t \wedge \tau_{R}^{n}}\left|\int_{0}^{s} \int_{Z}\left(u_{n}(r-), P_{n} g\left(s, u_{n}(s), z\right)\right) \tilde{\eta}(d r, d z)\right| \\
\leq & C_{1} \mathbb{E}\left[\int_{0}^{t \wedge \tau_{R}^{n}} \int_{Z}\left\|u_{n}(s)\right\|^{2}\left\|P_{n} g\left(s, u_{n}(s), z\right)\right\|^{2} \nu(d z) d s\right]^{\frac{1}{2}} \\
\leq & C_{1}\left[2 C_{1} \mathbb{E} \int_{0}^{t \wedge \tau_{R}^{n}}\left(k_{1}\left\|u_{n}(s)\right\|^{2}+k_{2}\left\|\nabla u_{n}(s)\right\|^{2}\right) d s\right]^{\frac{1}{2}} \times \\
& {\left[\frac{1}{2 C_{1}} \mathbb{E} \sup _{0 \leq s \leq t \wedge \tau_{R}^{n}}\left\|u_{n}(s)\right\|^{2}\right]^{\frac{1}{2}} }
\end{aligned}
$$




$$
\begin{aligned}
\leq & \frac{1}{2} \mathbb{E} \sup _{0 \leq s \leq t \wedge \tau_{R}^{n}}\left\|u_{n}(s)\right\|^{2}+2 C_{1}{ }^{2} k_{1} \mathbb{E} \int_{0}^{t \wedge \tau_{R}^{n}}\left\|u_{n}(s)\right\|^{2} d s \\
& +2 C_{1}{ }^{2} k_{2} \mathbb{E} \int_{0}^{t \wedge \tau_{R}^{n}}\left\|\nabla u_{n}(s)\right\|^{2} d s .
\end{aligned}
$$

Then we estimate the term $I_{3}$. Since

$$
\lambda_{1} \cdot \nabla\left(\left|u_{n}\right|^{2} u_{n}\right)+\left(\lambda_{2} \cdot \nabla u_{n}\right)\left|u_{n}\right|^{2}=\left(\left(2 \lambda_{1}+\lambda_{2}\right) \cdot \nabla u_{n}\right)\left|u_{n}\right|^{2}+\left(\lambda_{1} \cdot \nabla u_{n}\right) u_{n}^{2},
$$

we have

$$
\begin{aligned}
\mathbb{E} \sup _{0 \leq s \leq t \wedge \tau_{R}^{n}} I_{3}(s) \leq & \left(6\left|\lambda_{1}\right|+2\left|\lambda_{2}\right|\right) \mathbb{E} \sup _{0 \leq s \leq t \wedge \tau_{R}^{n}} \int_{0}^{s} \int_{D}\left|u_{n}(s, x)\right|^{3}\left|\nabla u_{n}(s, x)\right| d x d s \\
\leq & \left(6\left|\lambda_{1}\right|+2\left|\lambda_{2}\right|\right) \mathbb{E} \int_{0}^{t \wedge \tau_{R}^{n}}\left\|u_{n}(s)\right\|_{6}^{3}\left\|\nabla u_{n}(s)\right\| d s \\
\leq & \frac{1}{2} \mathbb{E} \int_{0}^{t \wedge \tau_{R}^{n}}\left\|\nabla u_{n}(s)\right\|^{2} d s+2\left(3\left|\lambda_{1}\right|+\left|\lambda_{2}\right|\right)^{2} \mathbb{E} \int_{0}^{t \wedge \tau_{R}^{n}}\left\|u_{n}(s)\right\|_{6}^{6} d s \\
\leq & \frac{1}{2} \mathbb{E} \int_{0}^{t \wedge \tau_{R}^{n}}\left\|\nabla u_{n}(s)\right\|^{2} d s+\frac{1}{2} \mathbb{E} \int_{0}^{t \wedge \tau_{R}^{n}}\left\|u_{n}(s)\right\|_{2 \sigma+2}^{2 \sigma+2} d s \\
& +C_{2}\left(\left|\lambda_{1}\right|,\left|\lambda_{2}\right|\right) \mathbb{E} \int_{0}^{t \wedge \tau_{R}^{n}}\left\|u_{n}(s)\right\|^{2} d s .
\end{aligned}
$$

In (3.6), we use the Young inequality in the last inequality as below

$$
\left\|u_{n}\right\|_{6}^{6} \leq\left\|u_{n}\right\|_{2 \sigma+2}^{\theta}\left\|u_{n}\right\|^{1-\theta} \leq \epsilon\left\|u_{n}\right\|_{2 \sigma+2}^{2 \sigma+2}+c(\epsilon)\left\|u_{n}\right\|^{2} .
$$

Putting (3.3)-(3.6) into (3.2), we have

$$
\begin{aligned}
& \frac{1}{2} \mathbb{E} \sup _{0 \leq s \leq t \wedge \tau_{R}^{n}}\left\|u_{n}(s)\right\|^{2}+\frac{C_{3}}{2} \mathbb{E} \int_{0}^{t \wedge \tau_{R}^{n}}\left\|\nabla u_{n}(s)\right\|^{2} d s+\frac{3}{2} \mathbb{E} \int_{0}^{t \wedge \tau_{R}^{n}}\left\|u_{n}(s)\right\|_{2 \sigma+2}^{2 \sigma+2} d s \\
\leq & \mathbb{E}\left\|u_{n}(0)\right\|^{2}+\frac{C_{4}}{2} \mathbb{E} \int_{0}^{t \wedge \tau_{R}^{n}}\left\|u_{n}(s)\right\|^{2} d s,
\end{aligned}
$$

where $\frac{C_{3}}{2}=\frac{3}{2}-\left(2 C_{1}^{2}+1\right) k_{2}, \frac{C_{4}}{2}=2 \gamma+\left(2 C_{1}^{2}+1\right) k_{1}+C_{2}\left(\left|\lambda_{1}\right|,\left|\lambda_{2}\right|\right)$. Therefore, if $k_{2}$ is small enough such that $\frac{3}{2}-\left(2 C_{1}^{2}+1\right) k_{2}>0$, we may apply Gronwall lemma to have

$$
\begin{aligned}
& \mathbb{E} \sup _{0 \leq s \leq t \wedge \tau_{R}^{n}}\left\|u_{n}(s)\right\|^{2}+\mathbb{E} \int_{0}^{t \wedge \tau_{R}^{n}}\left\|\nabla u_{n}(s)\right\|^{2} d s+\mathbb{E} \int_{0}^{t \wedge \tau_{R}^{n}}\left\|u_{n}(s)\right\|_{2 \sigma+2}^{2 \sigma+2} d s \\
\leq & C\left(\mathbb{E}\left\|u_{n}(0)\right\|^{2}+1\right) .
\end{aligned}
$$

Recall that $\tau_{R}^{n} \uparrow T$, and $\mathbb{P}\left\{\tau_{R}^{n}<T\right\}=0$ as $R \rightarrow \infty$. It follows from Fatou lemma that

$$
\begin{aligned}
& \mathbb{E} \sup _{0 \leq t \leq T}\left\|u_{n}(t)\right\|^{2}+\mathbb{E} \int_{0}^{T}\left\|\nabla u_{n}(s)\right\|^{2} d s+\mathbb{E} \int_{0}^{T}\left\|u_{n}(s)\right\|_{2 \sigma+2}^{2 \sigma+2} d s \\
\leq & \liminf _{n \rightarrow \infty} \mathbb{E}\left(\sup _{0 \leq s \leq t \wedge \tau_{R}^{n}}\left\|u_{n}(s)\right\|^{2}+\int_{0}^{t \wedge \tau_{R}^{n}}\left(\left\|\nabla u_{n}(s)\right\|^{2}+\left\|u_{n}(s)\right\|_{2 \sigma+2}^{2 \sigma+2}\right) d s\right)
\end{aligned}
$$




$$
\leq C\left(\mathbb{E}\left\|u_{n}(0)\right\|^{2}+1\right),
$$

which completes the proof of Lemma 3.1.

Lemma 3.2 Under the same assumptions as in Theorem 2.5, there exists a constant $C$ such that

$$
\begin{aligned}
& \sup _{n} \mathbb{E}\left(\sup _{0 \leq t \leq T}\left\|\nabla u_{n}(t)\right\|^{2}+\int_{0}^{T}\left\|\Delta u_{n}(s)\right\|^{2} d s+\int_{0}^{T} \int_{D}\left|u_{n}(s, x)\right|^{2 \sigma}\left|\nabla u_{n}(s, x)\right|^{2} d x d s\right) \\
\leq & C\left(\mathbb{E}\left\|\nabla u_{n}(0)\right\|^{2}+1\right) .
\end{aligned}
$$

Proof : We apply Itô formula to $\left\|\nabla u_{n}(t)\right\|^{2}$ and take the real part of both sides:

$$
\begin{aligned}
\left\|\nabla u_{n}(t)\right\|^{2}= & \left\|\nabla u_{n}(0)\right\|^{2}+2 \operatorname{Re} \int_{0}^{t}\left(\nabla u_{n}(s), \nabla P_{n} G\left(u_{n}(s)\right)\right) d s \\
& +2 \operatorname{Re} \int_{0}^{t}\left(\nabla u_{n}(s-), \nabla P_{n} g\left(s, u_{n}(s), z\right)\right) \tilde{\eta}(d s, d z) \\
& +\int_{0}^{t} \int_{Z}\left\|\nabla P_{n} g\left(s, u_{n}(s), z\right)\right\|^{2} \eta(d s, d z) \\
= & \left\|\nabla u_{n}(0)\right\|^{2}+I_{4}+I_{5}+I_{6},
\end{aligned}
$$

where

$$
\begin{aligned}
& I_{5}=-2 \operatorname{Re} \int_{0}^{t}\left(\triangle u_{n}(s), P_{n} g\left(s, u_{n}(s), z\right) \tilde{\eta}(d s, d z),\right. \\
& I_{6}=\int_{0}^{t} \int_{Z}\left\|\nabla P_{n} g\left(s, u_{n}(s), z\right)\right\|^{2} \eta(d s, d z)
\end{aligned}
$$

and

$$
\begin{aligned}
I_{4}= & -2 \operatorname{Re} \int_{0}^{t}\left(\triangle u_{n}(s),(1+i \alpha) \triangle u_{n}(s)-(1-i \beta)\left|u_{n}(s)\right|^{2 \sigma} u_{n}(s)+\gamma u_{n}(s)+P_{n} F\left(u_{n}(s)\right)\right) d s \\
= & -2 \int_{0}^{t}\left\|\triangle u_{n}(s)\right\|^{2} d s+2 \gamma \int_{0}^{t}\left\|\nabla u_{n}(s)\right\|^{2} d s-2 \operatorname{Re} \int_{0}^{t}\left(\triangle u_{n}(s), P_{n} F\left(u_{n}(s)\right)\right) d s \\
& +2 \operatorname{Re}(1+i \beta) \int_{0}^{t} \int_{D}\left|u_{n}(s)\right|^{2 \sigma} \bar{u}_{n}(s) \triangle u_{n}(s) d x d s \\
= & -2 \int_{0}^{t}\left\|\triangle u_{n}(s)\right\|^{2} d s+2 \gamma \int_{0}^{t}\left\|\nabla u_{n}(s)\right\|^{2} d s+I_{41}+I_{42} .
\end{aligned}
$$

Take the supremum and expectation in turn over the interval $[0, t]$ on both sides of (3.8), and then we estimate the last three items in the following:

$$
\begin{aligned}
\mathbb{E} \sup _{0 \leq s \leq t} I_{5}(s) & \leq 2 \mathbb{E}\left[\int_{0}^{t} \int_{Z}\left\|\nabla u_{n}(s)\right\|^{2}\left\|\nabla P_{n} g\left(s, u_{n}(s), z\right)\right\|^{2} \nu(d z) d s\right]^{\frac{1}{2}} \\
& \leq 2 \mathbb{E}\left[\int_{0}^{t} \int_{Z}\left\|\nabla u_{n}(s)\right\|^{2} \int_{D}\left|\partial_{u} P_{n} g\left(s, u_{n}(s, x), z\right)\right|^{2}\left|\nabla u_{n}(s, x)\right|^{2} d x \nu(d z) d s\right]^{\frac{1}{2}}
\end{aligned}
$$




$$
\begin{aligned}
\leq & 2 \mathbb{E}\left[\int_{0}^{t} \int_{Z}|h(z)|^{2} \nu(d z)\left\|\nabla u_{n}(s)\right\|^{2} \int_{D}\left|u_{n}(s, x)\right|^{2}\left|\nabla u_{n}(s, x)\right|^{2} d x d s\right]^{\frac{1}{2}} \\
\leq & 2\|h(z)\|_{L^{2}(Z, \nu)}^{2} \mathbb{E}\left[\int_{0}^{t}\left\|\nabla u_{n}(s)\right\|^{2} \int_{D}\left|u_{n}(s, x)\right|^{2}\left|\nabla u_{n}(s, x)\right|^{2} d x d s\right]^{\frac{1}{2}} \\
\leq & \frac{1}{2} \mathbb{E} \sup _{0 \leq s \leq t}\left\|\nabla u_{n}(s)\right\|^{2}+8\|h(z)\|_{L^{2}(Z, \nu)}^{4} \mathbb{E} \int_{0}^{t} \int_{D}\left|u_{n}(s, x)\right|^{2}\left|\nabla u_{n}(s, x)\right|^{2} d x d s \\
\leq & \frac{1}{2} \mathbb{E} \sup _{0 \leq s \leq t}\left\|\nabla u_{n}(s)\right\|^{2}+\epsilon_{1} \mathbb{E} \int_{0}^{t} \int_{D}\left|u_{n}(s)\right|^{2 \sigma}\left|\nabla u_{n}(s, x)\right|^{2} d x d s \\
& +C\left(\epsilon_{1},\|h\|_{L^{2}(Z, \nu)}\right) \mathbb{E} \int_{0}^{t}\left\|\nabla u_{n}(s)\right\|^{2} d s .
\end{aligned}
$$

The last inequality can be obtained as follows,

$$
\begin{aligned}
& \mathbb{E} \int_{0}^{t} \int_{D}\left|u_{n}(s, x)\right|^{2}\left|\nabla u_{n}(s, x)\right|^{2} d x d s \\
= & \mathbb{E} \int_{0}^{t} \int_{D}\left|u_{n}(s, x)\right|^{2}\left|\nabla u_{n}(s, x)\right|^{\frac{2}{\sigma}}\left|\nabla u_{n}(s, x)\right|^{2-\frac{2}{\sigma}} d x d s \\
\leq & \mathbb{E} \int_{0}^{t}\left[\int_{D}\left(\left|u_{n}(s, x)\right|^{2}\left|\nabla u_{n}(s, x)\right|^{\frac{2}{\sigma}}\right)^{\sigma} d x\right]^{\frac{1}{\sigma}}\left[\int_{D}\left(\left|\nabla u_{n}(s, x)\right|^{2-\frac{2}{\sigma}}\right)^{\frac{\sigma}{\sigma-1}} d x\right]^{\frac{\sigma-1}{\sigma}} d s \\
\leq & \epsilon \mathbb{E} \int_{0}^{t} \int_{D}\left|u_{n}(s, x)\right|^{2 \sigma}\left|\nabla u_{n}(s, x)\right|^{2} d x d s+C(\epsilon) \mathbb{E} \int_{0}^{t}\left\|\nabla u_{n}(s)\right\|^{2} d s .
\end{aligned}
$$

For the item $I_{6}$, we notice that

$$
\begin{aligned}
\mathbb{E} \sup _{0 \leq s \leq t} I_{6}(s) & =\mathbb{E} \int_{0}^{t} \int_{Z}\left\|\nabla P_{n} g\left(s, u_{n}(s), z\right)\right\|^{2} \eta(d s, d z) \\
& =\mathbb{E} \int_{0}^{t} \int_{Z} \int_{D}\left|\partial_{u} P_{n} g\left(s, u_{n}(s, x), z\right)\right|^{2}\left|\nabla u_{n}(s, x)\right|^{2} d x \nu(d z) d s, \\
\leq & \mathbb{E} \int_{0}^{t} \int_{D}\left[\int_{z}|h(z)|^{2} \nu(d z)\right]\left|u_{n}(s, x)\right|^{2}\left|\nabla u_{n}(s, x)\right|^{2} d x d s \\
\leq & \|h\|_{L^{2}(Z, \nu)}^{2} \mathbb{E} \int_{0}^{t} \int_{D}\left|u_{n}(s, x)\right|^{2}\left|\nabla u_{n}(s, x)\right|^{2} d x d s \\
\leq & \epsilon_{2} \mathbb{E} \int_{0}^{t} \int_{D}\left|u_{n}(s, x)\right|^{2 \sigma}\left|\nabla u_{n}(s, x)\right|^{2} d x d s \\
& +C\left(\epsilon_{2},\|h\|_{L^{2}(Z, \nu)}\right) \mathbb{E} \int_{0}^{t}\left\|\nabla u_{n}(s, x)\right\|^{2} d s .
\end{aligned}
$$

From (3.5), we can obtain

$$
\begin{aligned}
& \mathbb{E} \sup _{0 \leq s \leq t} I_{41}(s) \\
\leq & 2 \mathbb{E}\left|\int_{0}^{t} \int_{D}\left[\bar{\lambda}_{1} \cdot \nabla\left(\left|u_{n}(s, x)\right|^{2} \bar{u}_{n}(s, x)\right)+\left(\bar{\lambda}_{2} \cdot \nabla \bar{u}_{n}(s, x)\right)\left|u_{n}(s, x)\right|^{2}\right] \triangle u_{n}(s, x) d x d s\right|
\end{aligned}
$$




$$
\begin{aligned}
& \leq\left(6\left|\lambda_{1}\right|+2\left|\lambda_{2}\right|\right) \mathbb{E} \int_{0}^{t} \int_{D}\left|u_{n}(s, x)\right|^{2}\left|\nabla u_{n}(s, x)\right|\left|\triangle u_{n}(s, x)\right| d x d s \\
& \leq \frac{1}{2} \mathbb{E} \int_{0}^{t}\left\|\triangle u_{n}(s)\right\|^{2} d s+2\left(3\left|\lambda_{1}\right|+\left|\lambda_{2}\right|\right)^{2} \mathbb{E} \int_{0}^{t} \int_{D}\left|u_{n}(s, x)\right|^{4}\left|\nabla u_{n}(s, x)\right|^{2} d x d s \\
& =\frac{1}{2} \mathbb{E} \int_{0}^{t}\left\|\triangle u_{n}(s)\right\|^{2} d s+C\left(\left|\lambda_{1}\right|,\left|\lambda_{2}\right|\right) \mathbb{E} \int_{0}^{t} \int_{D}\left|u_{n}(s, x)\right|^{4}\left|\nabla u_{n}(s, x)\right|^{\frac{4}{\sigma}}\left|\nabla u_{n}(s, x)\right|^{2-\frac{4}{\sigma}} d x d s \\
& \leq \frac{1}{2} \mathbb{E} \int_{0}^{t}\left\|\triangle u_{n}(s)\right\|^{2} d s \\
& +C \mathbb{E} \int_{0}^{t}\left[\int_{D}\left(\left|u_{n}(s, x)\right|^{4}\left|\nabla u_{n}(s, x)\right|^{\frac{4}{\sigma}}\right)^{\frac{\sigma}{2}} d x\right]^{\frac{2}{\sigma}}\left[\int_{D}\left(\left|\nabla u_{n}(s, x)\right|^{2-\frac{4}{\sigma}}\right)^{\frac{\sigma}{\sigma-2}} d x\right]^{\frac{\sigma-2}{\sigma}} d s \\
& \leq \frac{1}{2} \mathbb{E} \int_{0}^{t}\left\|\triangle u_{n}(s)\right\|^{2} d s \\
& +\epsilon_{3} \mathbb{E} \int_{0}^{t} \int_{D}\left|u_{n}(s, x)\right|^{2 \sigma}\left|\nabla u_{n}(s, x)\right|^{2} d x+C\left(\epsilon_{3},\left|\lambda_{1}\right|,\left|\lambda_{2}\right|\right) \mathbb{E} \int_{0}^{t}\left\|\nabla u_{n}(s)\right\|^{2} d s .
\end{aligned}
$$

In order to estimate the term $I_{42}$, firstly we have

$$
\begin{gathered}
2 \operatorname{Re}(1+i \beta) \int_{D}\left|u_{n}(s, x)\right|^{2 \sigma} \bar{u}_{n}(s, x) \triangle u_{n}(s, x) d x \\
=-2 \operatorname{Re}(1+i \beta) \int_{D}\left|u_{n}(s, x)\right|^{2(\sigma-1)}\left[(\sigma+1)\left|u_{n}(s, x)\right|^{2}\left|\nabla u_{n}(s, x)\right|^{2}\right. \\
\left.+\sigma\left(\bar{u}_{n}(s, x)\right)^{2}\left(\nabla u_{n}(s, x)\right)^{2}\right] d x \\
=-\int_{D}\left|u_{n}(s, x)\right|^{2(\sigma-1)} \sum_{j=1}^{2}\left(\bar{u}_{n}(s, x) \partial_{j} u_{n}(s, x), u_{n}(s, x) \partial_{j} \bar{u}_{n}(s, x)\right) \\
\times M(\beta, \sigma)\left(\begin{array}{c}
u_{n} \partial_{j} \bar{u}_{n} \\
\bar{u}_{n} \partial_{j} u_{n}
\end{array}\right)(s, x) d x
\end{gathered}
$$

where

$$
M(\beta, \sigma)=\left(\begin{array}{cc}
\sigma+1 & (1-i \beta) \sigma \\
(1-i \beta) \sigma & \sigma+1
\end{array}\right)
$$

When $|\beta|<\frac{\sqrt{2 \sigma+1}}{\sigma}$, the matrix $M(\beta, \sigma)$ is definitely positive and thus the small eigenvalue $\lambda_{\beta}$ is positive. This gives

$$
2 \operatorname{Re}(1+i \beta) \int_{D}\left|u_{n}(s, x)\right|^{2 \sigma} \bar{u}_{n}(s, x) \triangle u_{n}(s, x) d x+2 \lambda_{\beta} \int_{D}\left|u_{n}(s, x)\right|^{2 \sigma}\left|\nabla u_{n}(s, x)\right|^{2} d x \leq 0 .
$$

Furthermore,

$$
\mathbb{E} \sup _{0 \leq s \leq t} I_{42}(s) \leq-2 \lambda_{\beta} \int_{0}^{t} \int_{D}\left|u_{n}(s, x)\right|^{2 \sigma}\left|\nabla u_{n}(s, x)\right|^{2} d x d s .
$$

Putting (3.9)-(3.12) into (3.8), we have

$$
\frac{1}{2} \mathbb{E} \sup _{0 \leq s \leq t}\left\|\nabla u_{n}(s)\right\|^{2}+\frac{3}{2} \mathbb{E} \int_{0}^{t}\left\|\triangle u_{n}(s)\right\|^{2} d s+\frac{C_{5}}{2} \mathbb{E} \int_{0}^{t} \int_{D}\left|u_{n}(s, x)\right|^{2 \sigma}\left|\nabla u_{n}(s, x)\right|^{2} d x d s
$$




$$
\leq \mathbb{E}\left\|\nabla u_{n}(0)\right\|^{2}+\frac{C_{6}}{2} \mathbb{E} \int_{0}^{t}\left\|\nabla u_{n}(s)\right\|^{2} d s,
$$

where $\frac{C_{5}}{2}=2 \lambda_{\beta}-\epsilon_{1}-\epsilon_{2}-\epsilon_{3}, \frac{C_{6}}{2}=C\left(\epsilon_{1}, \epsilon_{2}, \epsilon_{3},\left|\lambda_{1}\right|,\left|\lambda_{2}\right|,\|h\|_{L^{2}(Z, \nu)}\right)+2 \gamma$. Thus, choosing sufficiently small $\epsilon_{1}, \epsilon_{2}, \epsilon_{3}$ such that $2 \lambda_{\beta}-\epsilon_{1}-\epsilon_{2}-\epsilon_{3}>0$, we can apply Gronwall lemma to get

$$
\begin{aligned}
& \mathbb{E}\left(\sup _{0 \leq s \leq T}\left\|\nabla u_{n}(s)\right\|^{2}+\int_{0}^{T}\left\|\Delta u_{n}(s)\right\|^{2} d s+\int_{0}^{T} \int_{D}\left|u_{n}(s, x)\right|^{2 \sigma}\left|\nabla u_{n}(s, x)\right|^{2} d x d s\right) \\
\leq & C\left(\mathbb{E}\left\|\nabla u_{n}(0)\right\|^{2}+1\right) .
\end{aligned}
$$

Lemma 3.3 If $2 \leq p<2 \sigma$, there exists a constant $C$ such that

$$
\begin{aligned}
& \sup _{n} \mathbb{E}\left(\sup _{0 \leq s \leq t}\left\|\nabla u_{n}(s)\right\|^{p}+\int_{0}^{t}\left\|\nabla u_{n}(s)\right\|^{p-2}\left\|\Delta u_{n}(s)\right\|^{2} d s\right. \\
& \left.+\int_{0}^{t} \int_{D}\left\|\nabla u_{n}(s)\right\|^{p-2}\left|u_{n}(s, x)\right|^{2 \sigma}\left|\nabla u_{n}(s, x)\right|^{2} d x d s\right) \\
& \leq C \mathbb{E}\left(\left\|\nabla u_{n}(0)\right\|^{p}+1\right) .
\end{aligned}
$$

Proof : We apply Itô formula to $\left\|\nabla u_{n}(t)\right\|^{p}$ and take the real part on both sides to have

$$
\begin{aligned}
\left\|\nabla u_{n}(t)\right\|^{p}= & \left\|\nabla u_{n}(0)\right\|^{p}+p \operatorname{Re} \int_{0}^{t}\left\|\nabla u_{n}(s)\right\|^{p-2}\left(\nabla u_{n}(s), \nabla P_{n} G\left(u_{n}(s)\right)\right) d s \\
& +p \operatorname{Re} \int_{0}^{t} \int_{Z}\left\|\nabla u_{n}(s)\right\|^{p-2}\left(\nabla u_{n}(s-), \nabla P_{n} g\left(s, u_{n}(s), z\right)\right) \tilde{\eta}(d s, d z) \\
& +\operatorname{Re} \int_{0}^{t} \int_{Z}\left[\left\|\nabla\left(u_{n}(s-)+P_{n} g\left(s-, u_{n}(s-), z\right)\right)\right\|^{p}-\left\|\nabla u_{n}(s-)\right\|^{p}\right. \\
& \left.-p\left\|\nabla u_{n}(s-)\right\|^{p-2}\left(\nabla u_{n}(s-), \nabla P_{n} g\left(s-, u_{n}(s-), z\right)\right)\right] \eta(d s, d z) \\
= & \left\|\nabla u_{n}(0)\right\|^{p}+I_{7}+I_{8}+I_{9} .
\end{aligned}
$$

Then we estimate the last three items. For $I_{7}$, we have

$$
\begin{aligned}
I_{7}(s)= & -p \int_{0}^{t}\left\|\nabla u_{n}(s)\right\|^{p-2}\left(\triangle u_{n}(s), P_{n} G\left(u_{n}(s)\right)\right) d s \\
= & -p \int_{0}^{t}\left\|\nabla u_{n}(s)\right\|^{p-2}\left\|\triangle u_{n}(s)\right\|^{2} d s+p \gamma \int_{0}^{t}\left\|\nabla u_{n}(s)\right\|^{p} d s \\
& +p \operatorname{Re}(1+i \beta) \int_{0}^{t} \int_{D}\left\|\nabla u_{n}(s)\right\|^{p-2}\left|u_{n}(s, x)\right|^{2 \sigma} \bar{u}_{n}(s, x) \triangle u_{n}(s, x) d x d s \\
& -p \operatorname{Re} \int_{0}^{t}\left\|\nabla u_{n}(s)\right\|^{p-2}\left(\triangle u_{n}(s), P_{n} F\left(u_{n}(s)\right)\right) d s \\
= & -p \int_{0}^{t}\left\|\nabla u_{n}(s)\right\|^{p-2}\left\|\triangle u_{n}(s)\right\|^{2} d s+p \gamma \int_{0}^{t}\left\|\nabla u_{n}(s)\right\|^{p} d s+I_{71}+I_{72} .
\end{aligned}
$$

A similar estimation to the term $I_{41}$ and $I_{42}$ leads to

$$
I_{71}(s) \leq-2 \lambda_{\beta} \int_{0}^{t} \int_{D}\left\|\nabla u_{n}(s)\right\|^{p-2}\left|u_{n}(s, x)\right|^{2 \sigma}\left|\nabla u_{n}(s, x)\right|^{2} d x d s
$$


and

$$
\begin{aligned}
I_{72}(s) \leq & \frac{p}{2} \int_{0}^{t} \int_{D}\left\|\nabla u_{n}(s)\right\|^{p-2}\left\|\triangle u_{n}(s)\right\|^{2} d s+C\left(\epsilon_{4},\left|\lambda_{1}\right|,\left|\lambda_{2}\right|\right) \int_{0}^{t}\left\|\nabla u_{n}(s)\right\|^{p} d s \\
& +\epsilon_{4} \int_{0}^{t} \int_{D}\left\|\nabla u_{n}(s)\right\|^{p-2}\left|u_{n}(s, x)\right|^{2 \sigma}\left|\nabla u_{n}(s, x)\right|^{2} d x d s .
\end{aligned}
$$

Taking the supremum and expectation over the interval $[0, t]$ on both sides of (3.13), by (3.14) - (3.16) we obtain

$$
\begin{aligned}
\mathbb{E} \sup _{0 \leq s \leq t} I_{7}(s) \leq & -\frac{p}{2} \mathbb{E} \int_{0}^{t} \int_{D}\left\|\nabla u_{n}(s)\right\|^{p-2}\left\|\Delta u_{n}(s)\right\|^{2} d s+C_{7} \int_{0}^{t}\left\|\nabla u_{n}(s)\right\|^{p} d s \quad(3.17 \\
& +\left(-2 \lambda_{\beta}+\epsilon_{4}\right) \mathbb{E} \int_{0}^{t} \int_{D}\left\|\nabla u_{n}(s)\right\|^{p-2}\left|u_{n}(s, x)\right|^{2 \sigma}\left|\nabla u_{n}(s, x)\right|^{2} d x d s,
\end{aligned}
$$

where $C_{7}=C\left(\epsilon_{4},\left|\lambda_{1}\right|,\left|\lambda_{2}\right|\right)+p \gamma$. Next we will estimate the term $I_{8}$. Applying the Burkholder-Davis-Gundy inequality and the condition $\left(C_{3}\right)$, we get

$$
\begin{aligned}
\mathbb{E} \sup _{0 \leq s \leq t} I_{8}(s)= & p \mathbb{E} \sup _{0 \leq s \leq t}\left|\int_{0}^{s} \int_{Z}\left\|\nabla u_{n}(r)\right\|^{p-2}\left(\nabla u_{n}(r-), \nabla P_{n} g\left(r, u_{n}(r), z\right)\right) \tilde{\eta}(d r, d z)\right| \\
\leq & \bar{C} \mathbb{E}\left[\int_{0}^{t} \int_{Z}\left\|\nabla u_{n}(s)\right\|^{2 p-2}\left\|\nabla P_{n} g\left(s, u_{n}(s), z\right)\right\|^{2} \nu(d z) d s\right]^{\frac{1}{2}} \\
\leq & \bar{C}\|h(z)\|_{L^{2}(Z, \nu)} \mathbb{E}\left[\int_{0}^{t} \int_{D}\left\|\nabla u_{n}(s)\right\|^{2 p-2}\left|u_{n}(s, x)\right|^{2}\left|\nabla u_{n}(s, x)\right|^{2} d x d s\right]^{\frac{1}{2}} \\
\leq & \bar{C}\|h(z)\|_{L^{2}(Z, \nu)} \\
& \times \mathbb{E}\left[\int_{0}^{t} \int_{D}\left\|\nabla u_{n}(s)\right\|^{p}\left\|\nabla u_{n}(s)\right\|^{p-2}\left|u_{n}(s, x)\right|^{2}\left|\nabla u_{n}(s, x)\right|^{2} d x d s\right]^{\frac{1}{2}} \\
\leq & \frac{1}{2} \mathbb{E} \sup _{0 \leq s \leq t}\left\|\nabla u_{n}(s)\right\|^{p}+C \mathbb{E}_{0}^{t} \int_{D}\left\|\nabla u_{n}(s)\right\|^{p-2}\left|u_{n}(s, x)\right|^{2}\left|\nabla u_{n}(s, x)\right|^{2} d x d s \\
\leq & \left.\frac{1}{2} \mathbb{E} \sup _{0 \leq s \leq t}\left\|\nabla u_{n}(s)\right\|^{p}+C\left(\epsilon_{5}\right)\left\|\nabla u_{n}(s)\right\|^{2}\right) d s \\
& +C \mathbb{E} \int_{0}^{t}\left\|\nabla u_{n}(s)\right\|^{p-2}\left(\epsilon_{5} \int_{D}\left|u_{n}(s, x)\right|^{2 \sigma}\left|\nabla u_{n}(s, x)\right|^{2} d x\right. \\
\leq & \frac{1}{2} \mathbb{E} \sup _{0 \leq s \leq t}\left\|\nabla u_{n}(s)\right\|^{p}+C\left(\epsilon_{5},\|h(z)\|_{L^{2}(Z, \nu)}\right) \mathbb{E}_{0}^{t}\left\|\nabla u_{n}(s)\right\|^{p} d s \\
& +\epsilon_{5} \mathbb{E} \int_{0}^{t} \int\left\|\nabla u_{n}(s)\right\|^{p-2}\left|u_{n}(s, x)\right|^{2 \sigma}\left|\nabla u_{n}(s, x)\right|^{2} d x d s .
\end{aligned}
$$

On the other hand, by the Taylor formula, we have

$$
\left|\|x+h\|^{p}-\|x\|^{p}-p\|x\|^{p-2}(x, h)\right| \leq C_{p}\left(\|x\|^{p-2}\|h\|^{2}+\|h\|^{p}\right) \quad \text { for all } x, h \in H_{n} .
$$

Thus

$$
\mathbb{E} \sup _{0 \leq s \leq t} I_{9}(s)
$$




$$
\begin{aligned}
\leq & C \mathbb{E} \int_{0}^{t} \int_{Z}\left(\left\|\nabla u_{n}(s)\right\|^{p-2}\left\|\nabla P_{n} g\left(s, u_{n}(s), z\right)\right\|^{2} \nu(d z) d s\right. \\
& \left.+C \mathbb{E} \int_{0}^{t} \int_{Z}\left\|\nabla P_{n} g\left(s, u_{n}(s), z\right)\right\|^{p}\right) \nu(d z) d s \\
= & I_{91}(t)+I_{92}(t) .
\end{aligned}
$$

From the condition $\left(C_{3}\right)$ and (3.10), we deduce

$$
\begin{aligned}
I_{91}(t) \leq & C\left(\|h\|_{L^{2}(Z, \nu)}^{2}\right) \mathbb{E} \int_{0}^{t} \int_{D}\left\|\nabla u_{n}(s)\right\|^{p-2}\left|u_{n}(s, x)\right|^{2}\left|\nabla u_{n}(s, x)\right|^{2} d x d s \\
\leq & \epsilon_{6} \mathbb{E} \int_{0}^{t} \int_{D}\left\|\nabla u_{n}(s)\right\|^{p-2}\left|u_{n}(s, x)\right|^{2 \sigma}\left|\nabla u_{n}(s, x)\right|^{2} d x d s \\
& +C\left(\epsilon_{6},\|h(z)\|_{L^{2}(Z, \nu)}\right) \mathbb{E} \int_{0}^{t}\left\|\nabla u_{n}(s)\right\|^{p} d s .
\end{aligned}
$$

Now we estimate the last term of (3.19). By Young inequality, it follows that

$$
\begin{aligned}
& I_{92}(t)=\|h\|_{L^{p}(Z, \nu)}^{p} \mathbb{E} \int_{0}^{t}\left(\int_{D}\left|u_{n}(s, x)\right|^{2}\left|\nabla u_{n}(s, x)\right|^{2} d x\right)^{\frac{p}{2}} d s \\
& =C \mathbb{E} \int_{0}^{t}\left(\int_{D}\left|u_{n}(s, x)\right|^{2}\left|\nabla u_{n}(s, x)\right|^{\frac{4}{p}}\left|\nabla u_{n}(s, x)\right|^{2-\frac{4}{p}} d x\right)^{\frac{p}{2}} d s \\
& \leq C \mathbb{E} \int_{0}^{t}\left(\int_{D}\left(\left|u_{n}(s, x)\right|^{2}\left|\nabla u_{n}(s, x)\right|^{\frac{4}{p}}\right)^{\frac{p}{2}} d x\right) \\
& \left.\times\left(\int_{D}\left|\nabla u_{n}(s, x)\right|^{2-\frac{4}{p}}\right)^{\frac{p}{p-2}} d x\right)^{\frac{p-2}{2}} d s \\
& =C \mathbb{E} \int_{0}^{t}\left\|\nabla u_{n}(s)\right\|^{p-2} \int_{D}\left|u_{n}(s, x)\right|^{p}\left|\nabla u_{n}(s, x)\right|^{2} d x d s \\
& =C \mathbb{E} \int_{0}^{t}\left\|\nabla u_{n}(s)\right\|^{p-2} \int_{D}\left|u_{n}(s, x)\right|^{p}\left|\nabla u_{n}(s, x)\right|^{\frac{p}{\sigma}}\left|\nabla u_{n}(s, x)\right|^{2-\frac{p}{\sigma}} d x d s \\
& \leq \mathbb{E} \int_{0}^{t}\left\|\nabla u_{n}(s)\right\|^{p-2}\left(\epsilon_{7} \int_{D}\left|u_{n}(s, x)\right|^{2 \sigma}\left|\nabla u_{n}(s, x)\right|^{2} d x+C\left(\epsilon_{7}\right)\left\|\nabla u_{n}(s)\right\|^{2}\right) d s \\
& =\epsilon_{7} \mathbb{E} \int_{0}^{t} \int_{D}\left\|\nabla u_{n}(s)\right\|^{p-2}\left|u_{n}(s, x)\right|^{2 \sigma}\left|\nabla u_{n}(s, x)\right|^{2} d x d s \\
& +C\left(\epsilon_{7}\right) \mathbb{E} \int_{0}^{t}\left\|\nabla u_{n}(s)\right\|^{p} d s .
\end{aligned}
$$

Combining (3.17)-(3.21) with (3.13), we infer that

$$
\begin{aligned}
& \frac{1}{2} \mathbb{E} \sup _{0 \leq s \leq t}\left\|\nabla u_{n}(s)\right\|^{p}+\frac{p}{2} \mathbb{E} \int_{0}^{t}\left\|\nabla u_{n}(s)\right\|^{p-2}\left\|\triangle u_{n}(s)\right\|^{2} d s \\
&+\frac{C_{8}}{2} \mathbb{E} \int_{0}^{t} \int_{D}\left\|\nabla u_{n}(s)\right\|^{p-2}\left|u_{n}(s, x)\right|^{2 \sigma}\left|\nabla u_{n}(s, x)\right|^{2} d x d s \\
& \leq \mathbb{E}\left\|u_{n}(0)\right\|^{2}+\frac{C_{9}}{2} \mathbb{E} \int_{0}^{t}\left\|\nabla u_{n}(s)\right\|^{p} d s,
\end{aligned}
$$


where $\frac{C_{8}}{2}=2 \lambda_{\beta}-\sum_{i=4}^{7} \epsilon_{i}, \frac{C_{9}}{2}=p \gamma+C\left(\left|\lambda_{1}\right|,\left|\lambda_{2}\right|, \epsilon_{i}\right), i=4,5,6,7$. Thus, choosing sufficiently small $\epsilon_{i}$ such that $2 \lambda_{\beta}-\sum_{i=4}^{7} \epsilon_{i}>0$, we can use Gronwall lemma to get

$$
\begin{aligned}
& \mathbb{E}\left(\sup _{0 \leq s \leq T}\left\|\nabla u_{n}(s)\right\|^{p}+\int_{0}^{T} \int_{D}\left\|\nabla u_{n}(s)\right\|^{p-2}\left\|\triangle u_{n}(s)\right\|^{2} d s\right. \\
& \left.+\int_{0}^{T}\left\|\nabla u_{n}(s)\right\|^{p-2}\left|u_{n}(s, x)\right|^{2 \sigma}\left|\nabla u_{n}(s, x)\right|^{2} d x d s\right) \\
\leq & C\left(\mathbb{E}\left\|\nabla u_{n}(0)\right\|^{p}+1\right) .
\end{aligned}
$$

Since the constant $C$ is independent of $n$, the proof of Lemma 3.3 is complete.

Step 2 : We study the weak convergence of approximating sequences in this step. Under the same assumptions as in Theorem 2.5, there exists a subsequence of $\left\{u_{n}\right\}$, still denoted by $\left\{u_{n}\right\}$, satisfying the following Lemma:

Lemma 3.4 Let $T(u)=-(1-i \beta)|u|^{2 \sigma} u, s=(2 \sigma+2)^{*}=\frac{2 \sigma+2}{2 \sigma+1}, 2 \leq p<2 \sigma$. There exist processes $\tilde{u} \in L^{2}\left([0, T] \times \Omega ; H^{2}\right) \cap L^{p}\left(\Omega ; L^{\infty}([0, T] ; V)\right) \cap L^{2 \sigma+2}\left([0, T] \times \Omega ; L^{2 \sigma+2}(D)\right)$, $\tilde{T} \in L^{s}\left([0, T] \times \Omega ; L^{s}(D)\right), F \in L^{2}\left([0, T] \times \Omega ; H^{-2}\right), Y \in L^{2}\left([0, T] \times \Omega ; L^{2}(Z, \nu ; H)\right)$, where $H^{-2}$ is the dual space of $H^{2}$, such that

(1) $u_{n} \rightarrow \tilde{u}$ in $L^{2}\left([0, T] \times \Omega ; H^{2}\right) \cap L^{2 \sigma+2}\left([0, T] \times \Omega ; L^{2 \sigma+2}(D)\right)$;

(2) $u_{n}$ is weak star converging to $\tilde{u}$ in $L^{p}\left(\Omega ; L^{\infty}([0, T] ; V)\right)$;

(3) $P_{n} T\left(u_{n}\right) \rightarrow \tilde{T}$ in $L^{s}\left([0, T] \times \Omega ; L^{s}(D)\right)$;

(4) $P_{n} F\left(u_{n}\right) \rightarrow \tilde{F}$ in $L^{2}\left([0, T] \times \Omega ; H^{-2}\right)$;

(5) $P_{n} g\left(s, u_{n}(s), z\right) \rightarrow Y$ in $L^{2}\left([0, T] \times \Omega ; L^{2}(Z, \nu ; H)\right)$.

Proof : It is easy to see that (1) and (3) are straightforward consequences of Lemma 3.1. Since $L^{p}\left(\Omega ; L^{\infty}([0, T] ; V)\right) \cong\left(L^{p^{*}}\left(\Omega ; L^{1}\left([0, T] ; V^{*}\right)\right)\right)^{*}$, according to the Banach-Alaoglu theorem, we can get another weakly star convergent subsequence, still denoted by $\left\{u_{n}\right\}$, and $\tilde{u} \in L^{p}\left(\Omega ; L^{\infty}([0, T] ; V)\right)$ such that (2) holds. Moreover, for $v \in L^{2}\left([0, T] \times \Omega ; H^{2}\right)$, we find that

$$
\begin{aligned}
& \mathbb{E} \int_{0}^{T}\left\|P_{n} F\left(u_{n}(s)\right)\right\|_{H^{-2}}^{2} d s \\
& \leq 3\left(\left|\lambda_{1}\right|+\left|\lambda_{2}\right|\right) \sup _{\|v\|_{L^{2}\left([0, T] \times \Omega ; H^{2}\right)} \leq 1} \mathbb{E} \int_{0}^{T} \int_{D}\left|u_{n}(s, x)\right|^{2}\left|\nabla u_{n}(s, x) \| v(s, x)\right| d x d s \\
& \leq 3\left(\left|\lambda_{1}\right|+\left|\lambda_{2}\right|\right) \sup _{\|v\|_{L^{2}\left([0, T] \times \Omega ; H^{2}\right)} \leq 1} \mathbb{E} \int_{0}^{T} \int_{D}\left|u_{n}(s, x)\right|^{2}\left|\nabla u_{n}(s, x)\right|^{\frac{2}{\sigma}}\left|\nabla u_{n}(s, x)\right|^{1-\frac{2}{\sigma}} \\
& \times|v(s, x)| d x d s \\
& \leq 3\left(\left|\lambda_{1}\right|+\left|\lambda_{2}\right|\right) \sup _{\|v\|_{L^{2}\left([0, T] \times \Omega ; H^{2}\right)} \leq 1} \mathbb{E} \int_{0}^{T}\left(\int_{D}\left|u_{n}(s, x)\right|^{2 \sigma}\left|\nabla u_{n}(s, x)\right|^{2} d x\right)^{\frac{1}{\sigma}} \\
& \times\left(\int_{D}\left|\nabla u_{n}(s, x)\right|^{2} d x\right)^{\frac{\sigma-2}{2 \sigma}}\|v(s)\| d s \\
& \leq \sup _{\|v\|_{L^{2}\left([0, T] \times \Omega ; H^{2}\right)} \leq 1} \mathbb{E} \int_{0}^{T}\|v\|^{2} d s+\mathbb{E} \int_{0}^{T} \int_{D}\left|u_{n}(s, x)\right|^{2 \sigma}\left|\nabla u_{n}(s, x)\right|^{2} d x \\
& +C\left(\left|\lambda_{1}\right|,\left|\lambda_{2}\right|\right) \mathbb{E} \int_{0}^{T}\left\|\nabla u_{n}(s)\right\|^{2} d s<\infty,
\end{aligned}
$$


which implies that (4) holds. As for (5), by the condition $\left(C_{1}\right)$ and Lemma 3.1, we have

$$
\mathbb{E} \int_{0}^{T} \int_{Z}\left\|P_{n} g\left(s, u_{n}(s), z\right)\right\|^{2} \nu(d z) d s \leq \mathbb{E} \int_{0}^{T}\left(k_{1}\left\|u_{n}(s)\right\|^{2}+k_{2}\left\|\nabla u_{n}(s)\right\|^{2}\right) d s<\infty .
$$

So we konw that $P_{n} g$ has a subsequence converging weakly in $L^{2}\left([0, T] \times \Omega, L^{2}(Z, \nu ; H)\right)$ to $Y$.

Step 3 : Define $X:=(1+i \alpha) \triangle \tilde{u}+\tilde{T}+\gamma \tilde{u}+\tilde{F}$ and then a $V^{*}$-valued process $u$ by

$$
u(t):=u_{0}+\int_{0}^{t} X(s) d s+\int_{0}^{t} \int_{Z} Y(s, z) \tilde{\eta}(d z, d s)
$$

such that $u$ is a $V^{*}$-valued modification of the $V$-valued process $\tilde{u}$ in Lemma 3.4, i.e $u=\tilde{u}$ $d t \times \mathbb{P}$-a.e. in $V$. The aim of this step is to verify the following identities $d t \times \mathbb{P}$-a.e. on $[0, T] \times \Omega:$

$$
G(\tilde{u}(s))=X(s), \quad g(s, \tilde{u}, z)=Y(s, z) \quad \text { a.s. }
$$

Take $\phi$ from $L^{2}([0, T] \times \Omega ; V) \cap L^{2 \sigma+2}\left([0, T] \times \Omega ; L^{2 \sigma+2}(D)\right) \cap L^{p}\left(\Omega ; L^{\infty}([0, T] ; V)\right)$. Denote

$$
\begin{aligned}
& I=-(1-i \beta)\left\langle\left|u_{n}\right|^{2 \sigma} u_{n}-|\phi|^{2 \sigma} \phi, u_{n}-\phi\right\rangle, \\
& m=\operatorname{Re}\left\langle\left|u_{n}\right|^{2 \sigma} u_{n}-|\phi|^{2 \sigma} \phi, u_{n}-\phi\right\rangle, \quad n=\operatorname{Im}\left\langle\left|u_{n}\right|^{2 \sigma} u_{n}-|\phi|^{2 \sigma} \phi, u_{n}-\phi\right\rangle, \\
& F\left(u_{n}\right)=\left(\left(2 \lambda_{1}+\lambda_{2}\right) \cdot \nabla u_{n}\right)\left|u_{n}\right|^{2}+\left(\lambda_{1} \cdot \nabla u_{n}\right) u_{n}^{2}=F_{1}\left(u_{n}\right)+F_{2}\left(u_{n}\right), \\
& J=\left(F_{1}\left(u_{n}\right)-F_{1}(\phi), u_{n}-\phi\right)=\left(\left(2 \lambda_{1}+\lambda_{2}\right) \cdot\left(\left|u_{n}\right|^{2} \nabla u_{n}-|\phi|^{2} \nabla \phi\right), u_{n}-\phi\right), \\
& K=\left(F_{2}\left(u_{n}\right)-F_{2}(\phi), u_{n}-\phi\right)=\left(\lambda_{1} \cdot\left(u_{n}^{2} \nabla u_{n}-\phi^{2} \nabla \phi\right), u_{n}-\phi\right) .
\end{aligned}
$$

In order to obtain monotonicity properties of $G$, we need the following two lemmas.

Lemma 3.5 If $0<|\beta|<\frac{\sqrt{2 \sigma+1}}{\sigma}$, we have

$$
\operatorname{Re} I \leq-\left(1-\frac{\sigma}{\sqrt{2 \sigma+1}}|\beta|\right) 2^{-2 \sigma}\left\|u_{n}-\phi\right\|_{2 \sigma+2}^{2 \sigma+2} .
$$

Proof : Note that

$$
\begin{aligned}
m= & \operatorname{Re}\left\langle\left|u_{n}\right|^{2 \sigma} u_{n}-|\phi|^{2 \sigma} \phi, u_{n}-\phi\right\rangle \\
= & \operatorname{Re}\left\langle\left(\left|u_{n}\right|^{2 \sigma}-|\phi|^{2 \sigma}\right) u_{n}+|\phi|^{2 \sigma}\left(u_{n}-\phi\right), u_{n}-\phi\right\rangle \\
= & \int_{D}|\phi(s, x)|^{2 \sigma}\left|u_{n}(s, x)-\phi(s, x)\right|^{2} d x \\
& +\frac{1}{2} \int_{D}\left(\left|u_{n}(s, x)\right|^{2 \sigma}-|\phi(s, x)|^{2 \sigma}\right)\left(\left|u_{n}(s, x)\right|^{2}+\left|u_{n}(s, x)-\phi(s, x)\right|^{2}-|\phi(s, x)|^{2}\right) d x \\
\geq & \int_{D}|\phi(s, x)|^{2 \sigma}\left|u_{n}(s, x)-\phi(s, x)\right|^{2} d x \\
& +\frac{1}{2} \int_{D}\left(\left|u_{n}\right|^{2 \sigma}-|\phi(s, x)|^{2 \sigma}\right)\left|u_{n}(s, x)-\phi(s, x)\right|^{2} d x \\
= & \frac{1}{2} \int_{D}\left(\left|u_{n}(s, x)\right|^{2 \sigma}+|\phi(s, x)|^{2 \sigma}\right)\left|u_{n}(s, x)-\phi(s, x)\right|^{2} d x
\end{aligned}
$$




$$
\geq 2^{-2 \sigma} \int_{D}\left|u_{n}(s, x)-\phi(s, x)\right|^{2 \sigma+2} d x .
$$

Let $p=2 \sigma+2$, then $\frac{|n|}{m} \leq \frac{\sigma}{\sqrt{2 \sigma+1}}$. By Lemma 2.3, we find that

$$
\begin{aligned}
\operatorname{Re} I & =-m-\beta n \leq-\left(1-\frac{\sigma}{\sqrt{2 \sigma+1}}|\beta|\right) m \\
& \leq-\left(1-\frac{\sigma}{\sqrt{2 \sigma+1}}|\beta|\right) 2^{-2 \sigma}\left\|u_{n}-\phi\right\|_{2 \sigma+2}^{2 \sigma+2},
\end{aligned}
$$

if $0<|\beta|<\frac{\sqrt{2 \sigma+1}}{\sigma}$.

Lemma 3.6 We denote $w=u_{n}-\phi$ for simplicity, there exist enough small parameters $\tilde{\epsilon}, \hat{\epsilon}$ such that

$$
\begin{aligned}
\operatorname{Re} J \leq & \tilde{\epsilon}\|\nabla w\|_{2}^{2}+\hat{\epsilon}\|w\|_{2 \sigma+2}^{2 \sigma+2}+\left(C\left(\epsilon_{8}, \epsilon_{9}\right)+\left(\epsilon_{13}+\epsilon_{15}\right)\|\phi\|^{2}+C\left(\epsilon_{10}, \epsilon_{11}\right)\|\nabla \phi\|^{\frac{2 \sigma}{\sigma-1}}\right. \\
& \left.+C\left(\epsilon_{12}, \epsilon_{13}\right)\|\nabla \phi\|^{\frac{7 \sigma-2}{\sigma+1}}+C\left(\epsilon_{14}, \epsilon_{15}\right)\|\nabla \phi\|^{\frac{10 \sigma+4}{\sigma+4}}\right)\|w\|^{2} .
\end{aligned}
$$

Proof : First note that

$$
\begin{aligned}
\operatorname{Re} J & \leq C \int_{D}\left|\left(\left|u_{n}(s, x)\right|^{2} \nabla u_{n}(s, x)-|\phi(s, x)|^{2} \nabla \phi(s, x)\right) \bar{w}(s, x)\right| d x \\
& =C \int_{D} \mid\left(\left|u_{n}(s, x)\right|^{2} \nabla w(s, x)+u_{n}(s, x) \nabla \phi(s, x) \bar{w}(s, x)\right. \\
& \quad+\bar{\phi}(s, x) \nabla \phi(s, x) w(s, x)) \bar{w}(s, x) \mid d x \\
\leq & C \int_{D}\left(|w(s, x)|^{3}|\nabla w(s, x)|+|w(s, x)|^{3}|\nabla \phi(s, x)|+|w(s, x)|^{2}|\phi(s, x)||\nabla \phi(s, x)|\right. \\
& \left.\quad+|w(s, x)||\nabla w(s, x)||\phi(s, x)|^{2}\right) d x \\
= & J_{1}+J_{2}+J_{3}+J_{4} .
\end{aligned}
$$

In the following, we will estimate the four items $J_{1}, J_{2}, J_{3}, J_{4}$ by Sobolev embedding inequality, Hölder inequality and Young inequality. For $J_{1}$, we have

$$
\begin{aligned}
J_{1} & =\int_{D}|w(s, x)|^{3}|\nabla w(s, x)| d x \leq\|\nabla w\|\|w\|_{6}^{3} \leq \epsilon_{8}\|\nabla w\|^{2}+C\left(\epsilon_{8}\right)\|w\|_{6}^{6} \\
& \leq \epsilon_{8}\|\nabla w\|^{2}+\epsilon_{9}\|w\|_{2 \sigma+2}^{2 \sigma+2}+C\left(\epsilon_{8}, \epsilon_{9}\right)\|w\|^{2} .
\end{aligned}
$$

The last inequality of $(\underline{3.23})$ is due to (3.7). To estimate the second item $J_{2}$, we first notice

$$
\|w\|_{6} \leq\|w\|^{1-\theta}\|w\|_{2 \sigma+2}^{\theta}
$$

where $\theta=\frac{2 \sigma+2}{3 \sigma}$. From the Gagliardo-Nirenberg inequality for two-dimensional domain, we have

$$
\|w\|_{6} \leq\|w\|^{\frac{1}{3}}\|\nabla w\|^{\frac{2}{3}}
$$

Thus

$$
J_{2}=\int_{D}|w(s, x)|^{3}|\nabla \phi(s, x)| d x \leq\|w\|_{6}^{\frac{3}{2}}\|w\|_{6}^{\frac{3}{2}}\|\nabla \phi\|
$$




$$
\begin{aligned}
& \leq\left(\|w\|^{\frac{1}{3}}\|\nabla w\|^{\frac{2}{3}}\right)^{\frac{3}{2}}\|w\|^{\frac{\sigma-2}{2 \sigma}}\|w\|_{2 \sigma+2}^{\frac{2 \sigma+2}{2 \sigma}}\|\nabla \phi\| \\
& =\|\nabla w\|\|w\|_{2 \sigma+2}^{\frac{2 \sigma+2}{2 \sigma}}\|w\|^{\frac{\sigma-1}{\sigma}}\|\nabla \phi\| \\
& \leq \epsilon_{10}\|\nabla w\|^{2}+\epsilon_{11}\|w\|_{2 \sigma+2}^{2 \sigma+2}+C\left(\epsilon_{10}, \epsilon_{11}\right)\|\nabla \phi\|^{\frac{2 \sigma}{\sigma-1}}\|w\|^{2} .
\end{aligned}
$$

For the third item $J_{3}$ in (3.22), we deduce that

$$
\begin{aligned}
& J_{3}=\int_{D}|w(s, x)|^{2}\left|\phi(s, x)\|\nabla \phi \mid d x \leq\| \nabla \phi\|\| \phi\left\|_{\frac{8 \sigma-4}{\sigma-2}}\right\| w \|_{\frac{16 \sigma-8}{3 \sigma}}\right. \\
& \leq\|\nabla \phi\|\|\phi\|^{\frac{\sigma-2}{4 \sigma-2}}\|\nabla \phi\|^{\frac{3 \sigma}{4 \sigma-2}}\|w\|^{\frac{3 \sigma}{4 \sigma-2}}\|\nabla w\|^{\frac{5 \sigma-4}{4 \sigma-2}} \\
& \leq \epsilon_{12}\|\nabla w\|^{2}+C\left(\epsilon_{12}\right)\left(\|\nabla \phi\|^{\frac{7 \sigma-2}{4 \sigma-2}}\|\phi\|^{\frac{\sigma-2}{4 \sigma-2}}\right)^{\frac{8 \sigma-4}{3 \sigma}}\|w\|^{2} \\
& =\epsilon_{12}\|\nabla w\|^{2}+C\left(\epsilon_{12}\right)\|\nabla \phi\|^{\frac{2(7 \sigma-2)}{3 \sigma}}\|\phi\|^{\frac{2(\sigma-2)}{3 \sigma}}\|w\|^{2} \\
& \leq \epsilon_{12}\|\nabla w\|_{2}^{2}+\left(\epsilon_{13}\|\phi\|_{2}^{2}+C\left(\epsilon_{12}, \epsilon_{13}\right)\|\nabla \phi\|^{\frac{7 \sigma-2}{\sigma+1}}\right)\|w\|^{2} \text {, }
\end{aligned}
$$

where $2<\frac{7 \sigma-2}{\sigma+1}<2 \sigma$.

Now we turn to the last item $J_{4}$. It follows that

$$
\begin{aligned}
J_{4} & =\int_{D}|w(s, x)\|\nabla w(s, x)\| \phi|^{2} d x \leq\|\nabla w\|\|w\|_{\frac{3 \sigma}{\sigma+1}}\|\phi\|_{\frac{12 \sigma}{\sigma-2}}^{2} \\
& \leq\|\nabla w\|^{1+\frac{\sigma-2}{3 \sigma}}\|w\|^{\frac{2 \sigma+2}{3 \sigma}}\|\phi\|_{\frac{12 \sigma}{\sigma-2}}^{2} \\
& \leq \epsilon_{14}\|\nabla w\|^{2}+C\left(\epsilon_{14}\right)\|\phi\|_{\frac{12 \sigma}{\sigma-2}}^{\frac{6 \sigma}{\sigma+1}}\|w\|^{2} \\
& \leq \epsilon_{14}\|\nabla w\|^{2}+C\left(\epsilon_{14}\right)\left(\|\phi\|^{\frac{\sigma-2}{6 \sigma}}\|\nabla \phi\|^{\frac{5 \sigma+2}{6 \sigma}}\right)^{\frac{6 \sigma}{\sigma+1}}\|w\|^{2} \\
& =\epsilon_{14}\|\nabla w\|^{2}+C\left(\epsilon_{14}\right)\|\phi\|^{\frac{\sigma-2}{\sigma+1}}\|\nabla \phi\|^{\frac{5 \sigma+2}{\sigma+1}}\|w\|^{2} \\
& \leq \epsilon_{14}\|\nabla w\|^{2}+\left(\epsilon_{15}\|\phi\|^{2}+C\left(\epsilon_{14}, \epsilon_{15}\right)\|\nabla \phi\|^{\frac{10 \sigma+4}{\sigma+4}}\right)\|w\|^{2},
\end{aligned}
$$

where $2<\frac{10 \sigma+4}{\sigma+4}<2 \sigma$.

Based on (3.22) $-(3.26)$, we deduce that

$$
\begin{aligned}
\operatorname{Re} J \leq & \tilde{\epsilon}\|\nabla w\|^{2}+\hat{\epsilon}\|w\|_{2 \sigma+2}^{2 \sigma+2}+\left(C\left(\epsilon_{8}, \epsilon_{9}\right)+\left(\epsilon_{13}+\epsilon_{15}\right)\|\phi\|^{2}+C\left(\epsilon_{10}, \epsilon_{11}\right)\|\nabla \phi\|^{\frac{2 \sigma}{\sigma-1}}\right. \\
& \left.+C\left(\epsilon_{12}, \epsilon_{13}\right)\|\nabla \phi\|^{\frac{7 \sigma-2}{\sigma+1}}+C\left(\epsilon_{14}, \epsilon_{15}\right)\|\nabla \phi\|^{\frac{10 \sigma+4}{\sigma+4}}\right)\|w\|^{2}
\end{aligned}
$$

where $\tilde{\epsilon}=\epsilon_{8}+\epsilon_{10}+\epsilon_{12}+\epsilon_{14}$ and $\hat{\epsilon}=\epsilon_{9}+\epsilon_{11}$. This puts an end of the proof of Lemma 3.6.

The estimate of Re $K$ is similar to Lemma 3.6. From Lemma 3.5 and Lemma 3.6, we know

$$
\begin{aligned}
& \operatorname{Re}\left\langle P_{n} G\left(u_{n}\right)-P_{n} G(\phi), u_{n}-\phi\right\rangle \\
= & \operatorname{Re}\left[(1+i \alpha)\left\langle\triangle\left(u_{n}-\phi\right), u_{n}-\phi\right\rangle+\gamma\left\langle u_{n}-\phi, u_{n}-\phi\right\rangle+I+J+K\right] \\
= & (-1+\tilde{\epsilon})\left\|\nabla\left(u_{n}-\phi\right)\right\|^{2}+\left(-\left(1-\frac{\sigma}{\sqrt{2 \sigma+1}}|\beta|\right) 2^{-2 \sigma}+\hat{\epsilon}\right)\left\|u_{n}-\phi\right\|_{2 \sigma+2}^{2 \sigma+2} \\
& +\left(C\left(\epsilon_{8}, \epsilon_{9}, \gamma\right)+\left(\epsilon_{13}+\epsilon_{15}\right)\|\phi\|^{2}+C\left(\epsilon_{10}, \epsilon_{11}\right)\|\nabla \phi\|^{\frac{2 \sigma}{\sigma-1}}\right. \\
& \left.+C\left(\epsilon_{12}, \epsilon_{13}\right)\|\nabla \phi\|^{\frac{7 \sigma-2}{\sigma+1}}+C\left(\epsilon_{14}, \epsilon_{15}\right)\|\nabla \phi\|^{\frac{10 \sigma+4}{\sigma+4}}\right)\left\|\left(u_{n}-\phi\right)\right\|^{2} .
\end{aligned}
$$


Applying Itô formula to the process $e^{-r(t)}\left\|u_{n}(t)\right\|^{2}(r(t)$ will be defined later) and taking the real part, we obtain

$$
\begin{aligned}
e^{-r(t)}\left\|u_{n}(t)\right\|^{2}= & \left\|u_{n}(0)\right\|^{2}-\int_{0}^{t} e^{-r(s)} r^{\prime}(s)\left\|u_{n}(s)\right\|^{2} d s \\
& +2 \operatorname{Re} \int_{0}^{t} e^{-r(s)}\left\langle u_{n}(s), P_{n} G\left(u_{n}(s)\right)\right\rangle d s \\
& +2 \operatorname{Re} \int_{0}^{t} e^{-r(s)}\left(u_{n}(s-), P_{n} g\left(s, u_{n}(s), z\right)\right) \tilde{\eta}(d z, d s) \\
& +\int_{0}^{t} \int_{Z} e^{-r(s)}\left\|P_{n} g\left(s, u_{n}(s), z\right)\right\|^{2} \eta(d s, d z)
\end{aligned}
$$

Thus by taking the expectation on both sides of above equality, we have

$$
\begin{aligned}
\mathbb{E} & \left(e^{-r(t)}\left\|u_{n}(t)\right\|^{2}\right)-\mathbb{E}\left\|u_{n}(0)\right\|^{2} \\
=\mathbb{E} & \left(\operatorname { R e } \left(-\int_{0}^{t} e^{-r(s)} r^{\prime}(s)\left(2\left(u_{n}(s), \phi(s)\right)-\|\phi(s)\|^{2}\right) d s\right.\right. \\
& +2 \int_{0}^{t} e^{-r(s)}\left(\left\langle P_{n} G\left(u_{n}(s)\right)-P_{n} G(\phi(s)), \phi(s)\right\rangle+\left\langle P_{n} G(\phi(s)), u_{n}(s)\right\rangle\right) d s \\
& +\int_{0}^{t} \int_{Z} e^{-r(s)}\left(2\left(P_{n} g\left(s, u_{n}(s), z\right), P_{n} g(\phi(s))\right)-\left\|P_{n} g(s, \phi(s), z)\right\|^{2}\right) \nu(d z) d s \\
& -\int_{0}^{t} e^{-r(s)} r^{\prime}(s)\left\|u_{n}(s)-\phi(s)\right\|^{2} d s \\
& +2 \int_{0}^{t} e^{-r(s)}\left\langle P_{n} G\left(u_{n}(s)\right)-P_{n} G(\phi(s)), u_{n}(s)-\phi(s)\right\rangle d s \\
& \left.+\int_{0}^{t} \int_{Z} e^{-r(s)}\left\|P_{n} g\left(s, u_{n}(s), z\right)-P_{n} g(s, \phi(s), z)\right\|^{2} \nu(d z) d s\right) .
\end{aligned}
$$

According to the inequality (3.27) and the condition $\left(C_{2}\right)$, we can set

$$
\begin{aligned}
r(t)= & \int_{0}^{t}\left[2 \left(C\left(\epsilon_{8}, \epsilon_{9}, \gamma\right)+\left(\epsilon_{13}+\epsilon_{15}\right)\|\phi(s)\|^{2}+C\left(\epsilon_{10}, \epsilon_{11}\right)\|\nabla \phi(s)\|^{\frac{2 \sigma}{\sigma-1}}\right.\right. \\
& \left.\left.+C\left(\epsilon_{12}, \epsilon_{13}\right)\|\nabla \phi(s)\|^{\frac{7 \sigma-2}{\sigma+1}}+C\left(\epsilon_{14}, \epsilon_{15}\right)\|\nabla \phi(s)\|^{\frac{10 \sigma+4}{\sigma+4}}\right)+k_{3}\right] d s .
\end{aligned}
$$

Then we have

$$
\begin{aligned}
& -r^{\prime}(s)\left\|u_{n}(s)-\phi(s)\right\|^{2}+2 \operatorname{Re}\left\langle P_{n} G\left(u_{n}(s)\right)-P_{n} G(\phi(s)), u_{n}(s)-\phi(s)\right\rangle \\
& +\int_{Z}\left\|P_{n} g\left(s, u_{n}(s), z\right)-P_{n} g(s, \phi(s), z)\right\|^{2} \nu(d z) \\
\leq & \left(-2+2 \tilde{\epsilon}+k_{4}\right)\left\|\nabla\left(u_{n}(s)-\phi(s)\right)\right\|^{2}+2 K\left(\left\|\nabla\left(u_{n}(s)-\phi(s)\right)\right\|_{2 \sigma+2}^{2 \sigma+2}\right. \\
\leq & 0,
\end{aligned}
$$

provided that $\tilde{\epsilon}, \hat{\epsilon}, k_{4}$ are small enough such that $K=-\left(1-\frac{\sigma}{\sqrt{2 \sigma+1}}|\beta|\right) 2^{-2 \sigma}+\hat{\epsilon}<0$ and $-2+2 \tilde{\epsilon}+k_{4}<0$. Thus, 


$$
\begin{aligned}
& \mathbb{E} \operatorname{Re}\left(-\int_{0}^{t} e^{-r(s)} r^{\prime}(s)\left\|u_{n}(s)-\phi(s)\right\|^{2} d s\right. \\
& +2 \int_{0}^{t} e^{-r(s)}\left\langle P_{n} G\left(u_{n}(s)\right)-P_{n} G(\phi(s)), u_{n}(s)-\phi(s)\right\rangle d s \\
& \left.+\int_{0}^{t} \int_{Z} e^{-r(s)}\left\|P_{n} g\left(s, u_{n}(s), z\right)-P_{n} g(s, \phi(s), z)\right\|^{2} \nu(d z) d s\right) \leq 0 .
\end{aligned}
$$

Now we can rewrite (3.27) as below:

$$
\begin{aligned}
& \mathbb{E}\left(e^{-r(t)}\left\|u_{n}(t)\right\|^{2}\right)-\mathbb{E}\left\|u_{n}(0)\right\|^{2} \\
\leq & -\mathbb{E} \operatorname{Re} \int_{0}^{t} e^{-r(s)} r^{\prime}(s)\left(2\left(u_{n}(s), \phi(s)\right)-\|\phi(s)\|^{2}\right) d s \\
& +2 \mathbb{E} \operatorname{Re} \int_{0}^{t} e^{-r(s)}\left(\left\langle P_{n} G\left(u_{n}(s)\right)-P_{n} G(\phi(s)), \phi(s)\right\rangle+\left\langle P_{n} G(\phi(s)), u_{n}(s)\right\rangle\right) d s \\
& +\mathbb{E} \operatorname{Re} \int_{0}^{t} \int_{Z} e^{-r(s)}\left(2\left(P_{n} g\left(s, u_{n}(s), z\right), P_{n} g(s, \phi(s), z)\right)-\left\|P_{n} g(s, \phi(s), z)\right\|^{2}\right) \nu(d z) d s .
\end{aligned}
$$

By lower semi-continuity property of weak convergence and Lemma 3.5, we have

$$
\begin{aligned}
& \mathbb{E}\left(e^{-r(T)}\|u(T)\|^{2}-\left\|u_{n}(0)\right\|^{2}\right) \\
\leq & \liminf _{n \rightarrow \infty} \mathbb{E}\left(e^{-r(T)}\left\|u_{n}(T)\right\|^{2}-\left\|u_{n}(0)\right\|^{2}\right) \\
\leq & \liminf _{n \rightarrow \infty} \mathbb{E} \operatorname{Re}\left(\int_{0}^{T} e^{-r(s)} r^{\prime}(s)\left(\|\phi\|^{2}-2\left(u_{n}(s), \phi(s)\right)\right) d s\right. \\
& +2 \int_{0}^{T} e^{-r(s)}\left(\left\langle P_{n} G\left(u_{n}(s)\right)-P_{n} G(\phi(s)), \phi(s)\right\rangle+\left\langle P_{n} G(\phi(s)), u_{n}(s)\right\rangle\right) d s \\
& +\int_{0}^{T} \int_{Z} e^{-r(s)}\left(2\left(P_{n} g\left(s, u_{n}(s), z\right), P_{n} g(s, \phi(s), z)\right)-\left\|P_{n} g(s, \phi(s), z)\right\|^{2}\right) \nu(d z) d s \\
= & \mathbb{E} \operatorname{Re}\left[\int_{0}^{T} e^{-r(s)} r^{\prime}(s)\left(\|\phi(s)\|^{2}-2(\tilde{u}(s), \phi(s))\right) d s\right. \\
& +2 \int_{0}^{T} e^{-r(t)}(\langle X(s)-G(\phi(s)), \phi(s)\rangle+\langle G(\phi(s)), \tilde{u}(s)\rangle) d s \\
& \left.+\int_{0}^{T} \int_{Z} e^{-r(s)}\left(2(Y(s), g(s, \phi(s), z))-\|g(s, \phi(s), z)\|^{2}\right) \nu(d z) d s\right] .
\end{aligned}
$$

On the other hand, applying the Itô formula to the process $e^{r(T)}\|u(T)\|^{2}$, taking the real part and then taking expectation, we obtain

$$
\begin{aligned}
& \mathbb{E}\left(e^{-r(T)}\|u(T)\|^{2}-\left\|u_{n}(0)\right\|^{2}\right) \\
= & -\mathbb{E} \int_{0}^{T} e^{-r(s)} r^{\prime}(s)\|u(s)\|^{2} d s+2 \mathbb{E} \operatorname{Re} \int_{0}^{T} e^{-r(s)}\langle X(s), \tilde{u}(s)\rangle d s \\
& +\mathbb{E} \operatorname{Re} \int_{0}^{T} \int_{Z} e^{-r(s)}\|Y(s, z)\|^{2} \eta(d s, d z) .
\end{aligned}
$$


Therefore, in view of (3.30) and (3.31), we infer that

$$
\begin{aligned}
& \mathbb{E} \int_{0}^{T}\left(e ^ { - r ( s ) } \left[-r^{\prime}(s)\|\tilde{u}(s)-\phi(s)\|^{2}+2 \operatorname{Re}\langle X(s)-G(\phi(s)), \tilde{u}(s)-\phi(s)\rangle\right.\right. \\
& \left.\left.+\int_{Z}\|Y(s, z)-g(s, \phi(s), z)\|^{2} \nu(d z)\right]\right) d s \leq 0 .
\end{aligned}
$$

Taking $\phi=\tilde{u}$, we can obtain that $Y(s, z)=g(s, \tilde{u}(s), z)$ a.s. If we set $\phi=\tilde{u}-\epsilon v, v \in$ $L^{\infty}([0, T] \times \Omega, V), \epsilon>0$, it yields that

$$
\mathbb{E} \int_{0}^{T} e^{-r(s)}\left[-r^{\prime}(s) \epsilon^{2}\|v(s)\|^{2}+2 \epsilon \operatorname{Re}\langle X(s)-G(\tilde{u}(s)-\epsilon v(s)), v(s)\rangle\right] d s \leq 0 .
$$

Hence

$$
\mathbb{E} \int_{0}^{T} e^{-r(s)}\left[-r^{\prime}(s) \epsilon\|v(s)\|^{2}+2 \operatorname{Re}\langle X(s)-G(\tilde{u}(s)-\epsilon v(s)), v(s)\rangle\right] d s \leq 0 .
$$

Since $v$ is arbitrary, letting $\epsilon \rightarrow 0$, we get $X(s)=G(\tilde{u}(s))$ a.s. The proof of existence of the solution is complete.

Step 4 : It remains to prove the uniqueness of the solution to SPDE (2.3). Suppose $\omega(t)=u_{1}(t)-u_{2}(t)$, where $u_{1}, u_{2}$ are the solutions of (2.3) with initial conditions $u_{1}(0), u_{2}(0)$, respectively. We define the stopping time:

$$
\tau_{N}:=\inf \left\{t \geq 0,\left\|u_{1}(t)\right\|^{2} \geq N\right\} \wedge \inf \left\{t \geq 0,\left\|u_{2}(t)\right\|^{2} \geq N\right\} \wedge T .
$$

Applying Itô formula to $e^{-r\left(t \wedge \tau_{N}\right)}\left\|\omega\left(t \wedge \tau_{N}\right)\right\|^{2}$, taking the real part and then taking the expectation, we have

$$
\begin{aligned}
& e^{-r\left(t \wedge \tau_{N}\right)} \mathbb{E}\left(\left\|\omega\left(t \wedge \tau_{N}\right)\right\|^{2}-\left\|u_{1}(0)-u_{2}(0)\right\|^{2}\right) \\
= & -\mathbb{E} \int_{0}^{t \wedge \tau_{N}} e^{-r(s)} r^{\prime}(s)\|\omega(s)\|^{2} d s \\
& +2 \mathbb{E} \operatorname{Re} \int_{0}^{t \wedge \tau_{N}} e^{-r(s)}\left\langle G\left(u_{1}(s)\right)-G\left(u_{2}(s)\right), \omega(s)\right\rangle d s \\
& +\mathbb{E} \int_{0}^{t \wedge \tau_{N}} \int_{Z} e^{-r(s)}\left\|g\left(s, u_{1}(s), z\right)-g\left(s, u_{2}(s), z\right)\right\|^{2} \nu(d z) d s .
\end{aligned}
$$

It follows from (3.29) that

$$
\begin{aligned}
& e^{-r\left(t \wedge \tau_{N}\right)} \mathbb{E}\left(\left\|\omega\left(t \wedge \tau_{N}\right)\right\|^{2}-\left\|u_{1}(0)-u_{2}(0)\right\|^{2}\right) \\
= & \mathbb{E} \int_{0}^{t \wedge \tau_{N}} e^{-r(s)}\left[-r^{\prime}(s)\left\|u_{1}(s)-u_{2}(s)\right\|^{2}+2 \operatorname{Re}\left\langle G\left(u_{1}(s)\right)-G\left(u_{2}(s)\right), u_{1}-u_{2}\right\rangle\right. \\
& \left.+\int_{Z}\left\|g\left(s, u_{1}(s), z\right)-g\left(s, u_{2}(s), z\right)\right\|^{2} \nu(d z)\right] d s \\
\leq & 0,
\end{aligned}
$$

which implies the uniqueness of the solution and puts an end of the proof of Theorem 2.5. 


\section{Acknowledgements}

LL is supported in part by the NSF from Jiangsu province BK20171029 and the NSF of the Jiangsu Higher Education Committee of China No. 14KJB110016. HG is supported by a China NSF Grant No. 11531006, 11771123 and PAPD of Jiangsu Higher Education Institutions.

\section{References}

[1] Albeverio S., Brzeźniak Z., Wu J. L., Existence of global solutions and invariant measures for stochastic differential equations driven by Poisson type noise with non-Lipschitz coefficients, J. Math. Anal. Appl., 371 (2010), 309-322.

[2] Albeverio S., Rüdigeer B., Wu J. L., Analytic and probabilistic aspects of Lévy processes and fields in quantum theory, Lévy processes: Theory and Applications, edited by Barndorff-Nielsen O., Mikosch T., Resnick S., eds., Birkhäuser Verlag, Basel, 2001.

[3] Brzeźniak Z., Zhu J., Stochastic beam equations driven by compensated Poisson random measures, arXiv: 1011.5377.

[4] Brzeźniak Z., Hausenblas E., Zhu J., 2D stochastic Navier-Stokes equations driven by jump noise, Nonlinear Anal., 79 (2013), 122-139.

[5] Brzeźniak Z., Liu W., Strong solutions of SPDE with locally monotone coefficients driven by Lévy noise, Nonlinear Anal., 17 (2014), 283-310.

[6] Doelman A., "On the Nonlinear Evolution of Patterns Modulation Equations and Their Solutions", Ph.D. thesis, University of Utrech, 1990.

[7] Dong Z., Xu T. G., One-dimensional stochastic Burgers equation driven by Lévy processes, J. Funct. Anal., 243 (2007), 631-678.

[8] Dong Z., Xie Y. C., Global solutions of stochastic 2D Navier-Stokes equations with Lévy noise, Sci. China Ser. A 52 (2009), 1497-1524.

[9] Gao H. J., Bu C., Dirichlet inhomogeneous boundary value problem for the $n+1$ complex Ginzburg-Landau equation, J. Differential Equations, 198 (2004), 176-195.

[10] Gao H. J., Duan J. Q., On the initial-Value Problem for the Generalized TwoDimensional Ginzburg-Landau Equation, J. Math. Anal. Appl., 216 (1997), 536548.

[11] Gao H. J., Duan J.Q., Asymptotics for the generalized two-dimensional GinzburgLandau equation, J. Math. Anal. Appl., 247 (2000), 198-216.

[12] Ghidaglia J. M., Héron B., Dimension of the attractors associated to the GinzburgLandau equation, Phys. D, 28 (1987), 282-304. 
[13] Ginibre J., Velo G., The Cauchy problem in local spaces for the complex GinzurgLandau equation, Phys. D, 95 (1996), 191-228.

[14] Ichikawa A., Some inequalities for martingales and stochastic convolutions, Stoch. Anal. Appl., 4 (1986), 329-339.

[15] Li Y. S., Guo B. L., Global existence of solutions to the 2D Ginzburg-Landau equation, J. Math. Anal. Appl., 249 (2000), 412-432.

[16] Liu W., Röckner M., SPDE in Hilbert space with locally monotone coefficients, J. Funct. Anal., 259 (2010), 2902-2922.

[17] Mtivier M., Semimartingales: a course on stochastic processes, in: de Gruyter Studies in Mathematics, vol. 2, Walter de Gruyter Co., BerlinCNew York, 1982.

[18] Okazawa N., Yokota T., Global existence and smoothing effect for the complex Ginzburg-Landau equation with p-laplacian, J. Differential Equations, 182 (2002), 514-576.

[19] Peszat S., Zabczyk J., Stochastic partial differential equations with Lévy noise, Cambridge University Press, 2007.

[20] Prevot C., Rockner M., A concise course on stochastic partial differential equations, Lecture Notes on Mathematics, Springer, 2007.

[21] Röckner M., Zhang T. S., Stochastic evolutions of jump type: existence, uniqueness and large deviation principles, Potential Anal., 26 (2007), 255-279.

[22] Sun C. F., Gao H. J., Well-posedness for the stochastic 2D Primitive equations with Lévy noise, Sci. China Math., 56 (2013), 1629-1645.

[23] Shlesinger M. F., Zavslavsky G. M., Feisch U., eds., Lévy flights and related topics in physics, Springer-Verlag, 1995.

[24] Temam R., Infinite-Dimensional Systems in Mechanics and Physics, SpringerVerlag, New York, 1988.

[25] Wang G. L., Guo B. L., The asymptotic behavior of the stochastic Ginzburg-Landau equation with additive noise, Appl. Math. Comput., 198 (2008), 849-857.

[26] Yang D. S., The asymptotic behavior of the stochastic Ginzburg-Landau equation with multiplicative noise, J. Math. Phys., 45 (2004), 4064-4076.

[27] Yang D. S., On the generalized 2-D stochastic Ginzburg-Landau equation, Acta Math. Sin., 26 (2010), 1601-1612.

[28] Yang D. S., Large deviations for the stochastic derivative Ginzburg-Landau equation with multiplicative noise, Phys. D, 237 (2008), 82-91.

[29] Zhu J., A study of SPDEs w.r.t. compensated Poisson random measures and related topics, Ph.D. thesis, University of York, 2010. 